\title{
ACTIONS OF LOOP GROUPS ON HARMONIC MAPS
}

\author{
M. J. BERGVELT AND M. A. GUEST
}

\begin{abstract}
We describe a general framework in which subgroups of the loop group $\Lambda G l_{n} \mathbb{C}$ act on the space of harmonic maps from $S^{2}$ to $G l_{n} \mathbb{C}$. This represents a simplification of the action considered by Zakharov-Mikhailov-Shabat [ZM, ZS] in that we take the contour for the Riemann-Hilbert problem to be a union of circles; however, it reduces the basic ingredient to the well-known Birkhoff decomposition of $\Lambda G l_{n} \mathbb{C}$, and this facilitates a rigorous treatment. We give various concrete examples of the action, and use these to investigate a suggestion of Uhlenbeck [Uh] that a limiting version of such an action ("completion") gives rise to her fundamental process of "adding a uniton". It turns out that this does not occur, because completion preserves the energy of harmonic maps. However, in the special case of harmonic maps from $S^{2}$ to complex projective space, we describe a modification of this completion procedure which does indeed reproduce "adding a uniton".
\end{abstract}

One aspect of the theory of symmetry groups for differential equations is the idea of "proliferation of solutions". By this is meant that, starting with an obvious solution, application of symmetries can lead to further (perhaps less obvious) solutions. In this paper we shall apply the general framework described by Zakharov et al. [ZM, ZS] to the harmonic map equation (the principal chiral or sigma model of mathematical physics).

To put this into a wider context, we recall that a fundamental discovery in the study of the Korteweg-de Vries and related equations was that the space of solutions admits a certain infinite-dimensional symmetry group. Historically, the first manifestation of this (via Noether's theorem) was the appearance of infinitely many conservation laws. Later this led to an elegant algebraic description of solutions of the equation as the points of an orbit of a representation of an infinite-dimensional group. (See, for example, [Wi] for a brief description of some of these ideas.) The existence of previously unnoticed symmetries ("hidden symmetries") for other equations became an interesting possibility, and attempts have been made (so far with much less success than in the case of the $\mathrm{KdV}$ equation) to unearth them. One such example is the harmonic map equation for maps from a surface $D$ into a compact Lie group $G$ (or homogeneous space $G / H)$.

The basic observation [Po, Uh, ZM, ZS] which introduces an infinite-dimensional group is that harmonic maps from $D$ to $G$ correspond to certain

Received by the editors February 9, 1990.

1980 Mathematics Subject Classification (1985 Revision). Primary 58E20. 
holomorphic maps from $D$ to the based loop group $\Omega G$. By definition, $\Omega G$ is the infinite-dimensional Lie group consisting of all smooth maps from the circle $S^{1}$ (the unit complex numbers) into $G$ which send 1 to the identity element of $G$. Given the reformulation of the harmonic map equation in terms of $\Omega G$-valued maps, it is hardly surprising that the loop group $\Omega G$ can be made to act on the space of harmonic maps. However, it is not the naive "translation" action which is of interest, but a more complicated procedure involving an identification of $\Omega G$ with a homogeneous space of the group of loops in the complexified group $G^{c}$.

The action was introduced in general terms in [ZM, ZS], via the classical Riemann-Hilbert problem. The physics literature is extensive, and deals mainly with the infinitesimal action of the Lie algebra of $\Omega G$ (essentially the associated affine Kac-Moody Lie algebra) on the hypothetical tangent space to the space of harmonic maps. See, for example, [AJS, JS] and the references therein. From the point of view of analysis and harmonic maps, Uhlenbeck's paper [Uh] contains the first mathematical treatment of the group action. This, together with [ZM, ZS], provided the motivation for the present article. Our intention was, first, to set up the action in as elementary a way as possible, emphasizing the loop group and its so-called Birkhoff decomposition as the basic tool. This has the advantage that the Birkhoff factorization is widely known and easy to describe, and so we avoid a tiresome excursion into the delicate theory of the general Riemann-Hilbert problem. The Birkhoff decomposition is the link between the formal algebraic approach of the physicists and the contour integral approach of [Uh, ZM, ZS]. Second, we aimed to apply this theory to some concrete examples.

In $\S \S 1-5$ we set up a general theory for "dressing pseudo-actions" on harmonic maps, which includes essentially all the known situations. We prove that the action preserves energy of harmonic maps on the two-sphere (Propositions 3.3, 4.5). By a result of Atiyah and Donaldson (see $\$ 6$ ) this forces immediately the "collapsing" of the orbits to finite dimensions, a phenomenon which has been observed for different reasons by other authors (e.g. [AJS, Uh]). We also relate the rather complicated formula for the action to the much simpler operation of left multiplication (Theorem 5.3).

The main applications are given in $\S \S 6$ and 7 . We give a number of examples, based on the only example which was previously known, namely Theorem 5.3 of [Uh]. Part of the difficulty in constructing examples is the highly discontinuous nature of the action. In $\S 6$, motivated again by Uhlenbeck's example, we define a procedure of "completion", in which this discontinuity is exploited to enlarge orbits of the action. In $\S 7$, we investigate a suggestion of [Uh] that this sort of completion reproduces the fundamental procedure of "adding a uniton". Unfortunately the results (see Proposition 7.4 and Example 7.5) are negative: unitons cannot be added this way.

For the special example of harmonic maps from $S^{2}$ to $\mathbb{C} P^{n}$, we are able to describe explicitly all operations produced by the completion procedure. The 
classification of harmonic maps in this case is relatively straightforward (see [EL]). Using these results we show that a modification of the completion procedure of $\S 6$ does in fact allow us to "reduce" a general harmonic map to a constant map, thereby exhibiting a connection between the dressing pseudo-action and adding a uniton (Theorem 7.7).

It will be clear that this paper owes much to the stimulating articles [Uh, ZM, ZS]. We have had very helpful discussions with Y. Ohnita. This work began when both authors enjoyed the hospitality of the Max-Planck-Institut für Mathematik, and the second author gladly acknowledges the support of the Alexander von Humboldt-Stiftung during this period.

\section{HARMONIC MAPS AND EXTENDED SOLUTIONS}

Let $D$ be a connected and simply connected region of $\mathbb{C} \cup \infty=S^{2}$. We use $z$ as a local coordinate on $D, d z$ denotes the corresponding 1 -form on $D$, and $Z$ is the dual vector field. A map $\phi: D \rightarrow G l_{n} \mathbb{C}$ is harmonic if and only if it satisfies the equation

$$
Z \cdot\left(\bar{Z} \cdot \phi \phi^{-1}\right)+\bar{Z} \cdot\left(Z \cdot \phi \phi^{-1}\right)=0,
$$

where $Z \cdot \phi$ denotes the directional dervivative $\partial \phi / \partial z$ of the matrix-valued function $\phi$. For computational purposes it is useful to observe that $Z \cdot \phi=Z \phi-$ $\phi Z$ as operators on $\mathbb{C}^{n}$-valued functions, i.e., if $v: D \rightarrow \mathbb{C}^{n}$ then $(Z \cdot \phi) v=Z \cdot(\phi v)-\phi(Z \cdot v)$. For example, the harmonic map equation may be written in the expanded form

$$
\phi \bar{Z} \phi^{-1} Z+\phi Z \phi^{-1} \bar{Z}=Z \phi \bar{Z} \phi^{-1}+\bar{Z} \phi Z \phi^{-1} .
$$

For background information on this formulation of the harmonic map equation we refer to $\S 4$ of [Gu2].

Let

$$
\Lambda G l_{n} \mathbb{C}=\left\{\gamma: S^{1} \rightarrow G l_{n} \mathbb{C} \mid \gamma \text { smooth }\right\},
$$

and

$$
\Omega G l_{n} \mathbb{C}=\left\{\gamma \in \Lambda G l_{n} \mathbb{C} \mid \gamma(1)=I\right\} .
$$

These are called the free loop group and the based loop group (of $G l_{n} \mathbb{C}$ ), respectively. We consider $S^{1}$ as $\{\lambda \in \mathbb{C}|| \lambda \mid=1\}$. A map $\Phi: D \rightarrow \Omega G l_{n} \mathbb{C}$ is called (following [Uh]) an extended solution if and only if it satisfies a "linear system" of the form

$$
Z \cdot \Phi \Phi^{-1}=\frac{1}{2}(1-1 / \lambda) A, \quad \bar{Z} \cdot \Phi \Phi^{-1}=\frac{1}{2}(1-\lambda) B
$$

for some (smooth) $A, B: D \rightarrow \mathfrak{g l}_{n} \mathbb{C}$.

Proposition 1.1. (1) If $\Phi$ is an extended solution, then the map $\phi$ defined by $\phi(z)=\Phi(z)(-1)$ is harmonic.

(2) If $\phi$ is a harmonic map, and $g \in \Omega G l_{n} \mathbb{C}$, then there exists a unique extended solution $\Phi$ with the properties (a) $\phi(z)=\Phi(z)(-1)$ and (b) $\Phi(*)=g$, where * is some fixed basepoint of $D$. 
Proof. (1) Multiplying the linear system on the right by $\Phi$ and then differentiating, we obtain $(1-1 / \lambda) \bar{Z} \cdot(A \Phi)=2 \bar{Z} \cdot(Z \cdot \Phi)=2 Z \cdot(\bar{Z} \cdot \Phi)=(1-\lambda) Z \cdot(B \Phi)$. Now, $\bar{Z} \cdot(A \Phi)=\bar{Z} \cdot A \Phi+A \bar{Z} \cdot \Phi=\bar{Z} \cdot A \Phi+\frac{1}{2}(1-\lambda) A B \Phi$ and similarly $Z \cdot(B \Phi)=Z \cdot B \Phi+\frac{1}{2}(1-1 / \lambda) B A \Phi$. Substituting, and equating to zero the coefficient of each power of $\lambda$, we obtain the three equations

$$
\begin{gathered}
\bar{Z} \cdot A-Z \cdot B+[A, B]=0, \\
2 Z \cdot B=[A, B], \quad 2 \bar{Z} \cdot A=[B, A] .
\end{gathered}
$$

Adding the last two gives the harmonic map equation $\bar{Z} \cdot A+Z \cdot B=0$, as required.

(2) Consider the family of $\mathfrak{g l}_{n} \mathbb{C}$-valued complex 1 -forms on $D$ defined by

$$
\omega_{\lambda}=\frac{1}{2}(1-1 / \lambda) Z \cdot \phi \phi^{-1} d z+\frac{1}{2}(1-\lambda) \bar{Z} \cdot \phi \phi^{-1} d \bar{z}
$$

parametrized by complex numbers $\lambda$. Let $A=Z \cdot \phi \phi^{-1}, B=\bar{Z} \cdot \phi \phi^{-1}$. Observe that $d \omega_{\lambda}-\frac{1}{2}\left[\omega_{\lambda} \wedge \omega_{\lambda}\right]=\left(\omega_{1} / \lambda+\omega_{2}+\lambda \omega_{3}\right) d z \wedge d \bar{z}$, where $\omega_{1}=\bar{Z} \cdot A / 2+$ $[A, B] / 4, \omega_{2}=-\bar{Z} \cdot A / 2+Z \cdot B / 2-[A, B] / 2$, and $\omega_{3}=-Z \cdot B / 2+[A, B] / 4$. As $\phi$ is harmonic, we have $\bar{Z} \cdot A+Z \cdot B=0$. We also have the equation $\bar{Z} \cdot A-Z \cdot B+[A, B]=0$. (This is seen to be an identity on substituting the definitions of $A, B$; alternatively one may recognize it as the Maurer-Cartan equation.) It follows that $\omega_{1}=\omega_{2}=\omega_{3}=0$ (cf. the proof of part (1)). Thus the 1 -form $\omega_{\lambda}$ defines a flat connection on $D \times G l_{n} \mathbb{C}$. Since $D$ is simply connected, we may write $\omega_{\lambda}=d \Phi_{\lambda} \Phi_{\lambda}^{-1}$ for some $\Phi_{\lambda}: D \rightarrow G l_{n} \mathbb{C}$ (" $\omega_{\lambda}$ is gauge equivalent to the trivial connection"). The proof is completed by defining $\Phi(z)(\lambda)=\Phi_{\lambda}(z)$; condition (a) is immediate, and since $\Phi$ is only defined up to right translation, we may choose it so that condition (b) is satisfied. Since $\omega_{1}=0, \Phi(z)(1)$ is constant, hence it is equal to $\Phi(*)(1)=g(1)=I$. This shows that $\Phi$ takes values in $\Omega G l_{n} \mathbb{C}$.

Remarks. 1. If we take $g$ to be the restriction of a $G l_{n} \mathbb{C}$-valued function on a subset $U$ of $\mathbb{C}^{*}$, then $\Phi(z)$ will also be defined on $U$ (for all $z \in D$ ), rather than just on the unit circle. Moreover, $\Phi(z): U \rightarrow G l_{n} \mathbb{C}$ will be holomorphic if $g: U \rightarrow G l_{n} \mathbb{C}$ is holomorphic. An important example of this is the case $g=I$ (the identity loop); we obtain $\Phi(z): \mathbb{C}^{*} \rightarrow G l_{n} \mathbb{C}$ for all $z \in D$. Such an extended solution (i.e., with $\Phi(*)=I$ ) will be called a based extended solution.

2. If $\Phi$ takes values in $\Omega U_{n}$ (equivalently, if $\phi$ takes values in $U_{n}$ ), the equations

$$
Z \cdot \Phi \Phi^{-1}=\frac{1}{2}(1-1 / \lambda) A, \quad \bar{Z} \cdot \Phi \Phi^{-1}=\frac{1}{2}(1-\lambda) B
$$

are equivalent. This situation will be referred to as "the real case". The condition for $\Phi$ to be real is $\Phi(z)(\lambda)^{*}=\Phi(z)(\lambda)^{-1}$, for all $z \in D$ and for all $\lambda$ with $|\lambda|=1$. For a based extended solution, this is equivalent to $\Phi(z)(1 / \bar{\lambda})^{*}=\Phi(z)(\lambda)^{-1}$, for all $z \in D$ and $\lambda \in \mathbb{C}^{*}$.

3. In the real case, an extended solution is in particular a holomorphic map from $D$ to $\Omega U_{n}$, if $\Omega U_{n}$ is given the complex structure (see 8.9 of [PS]) for 
which $T_{1,0} \Omega U_{n}$ is spanned by elements of the form $X \lambda^{-i}$, for $X \in \mathfrak{g l}_{n} \mathbb{C}, i>$ 0 . This is because the equations for an extended solution $\Phi$ imply immediately that $d \Phi(Z) \subseteq T_{1,0} \Omega U_{n}$.

Example 1.2. For any map $f: D \rightarrow \mathrm{Gr}_{k}\left(\mathbb{C}^{n}\right)$ into the Grassmannian of $k$ planes in $\mathbb{C}^{n}$, let $\pi_{f}: D \rightarrow \mathfrak{g l}_{n} \mathbb{C}$ be the map which assigns to $z \in D$ the matrix of the orthogonal projection onto the $k$-plane $f(z)$. Similarly, let $\pi_{f}^{\perp}(z)$ be orthogonal projection onto $f(z)^{\perp}$. Then we obtain a map

$$
\phi=\pi_{f}^{\perp}-\pi_{f}: D \rightarrow U_{n} .
$$

This map $\phi$ is harmonic if and only if $f$ is harmonic, since the embedding $X \mapsto \pi_{X}^{\perp}-\pi_{X}$ is a totally geodesic embedding of $\mathrm{Gr}_{k}\left(\mathbb{C}^{n}\right)$ into $U_{n}$ (see $\S 4$ of [EL]). The harmonic map equation for $\phi$ is

$$
\pi_{f}^{\perp} Z \pi_{f}^{\perp} \bar{Z} \pi_{f}=\pi_{f}^{\perp} \bar{Z} \pi_{f} Z \pi_{f},
$$

where each of $\pi_{f}, \pi_{f}^{\perp}, Z, \bar{Z}$ are now considered as operators on $\mathbb{C}^{n}$-valued functions (see [Gu2]). Now, $f$ is a holomorphic map if and only if

$$
\pi_{f}^{\perp} \bar{Z} \pi_{f}=0 .
$$

Hence, if $f$ is holomorphic, it is certainly harmonic. One checks easily that an extended solution-not necessarily based-corresponding to $\phi$ is

$$
\Phi(z)(\lambda)=\pi_{f(z)}^{\perp}+\lambda \pi_{f(z)} .
$$

Conversely, given any map $f$, if the map $\Phi$ defined by $(*)$ is an extended solution, then $f$ must be holomorphic. Similarly, antiholomorphic maps $f: D \rightarrow$ $\mathrm{Gr}_{k}\left(\mathbb{C}^{n}\right)$ (namely, those for which $\pi_{f}^{\perp} Z \pi_{f}=0$ ) correspond to extended solutions $\Phi(z)(\lambda)=\pi_{f(z)}^{\perp}+\lambda^{-1} \pi_{f(z)}$.

We conclude this section with a remark on the energy functional, in the case where $D=S^{2}$.

Proposition 1.3. Let $\Phi: S^{2} \rightarrow \Omega G l_{n} \mathbb{C}$ be an extended solution with associated harmonic map $\phi: S^{2} \rightarrow G l_{n} \mathbb{C}$. Then the (normalized) energy $E(\phi)$ of $\phi$ is equal to the degree $\operatorname{deg} \Phi$ of $\Phi$.

Proof. The energy is defined by $E(\phi)=\int_{S^{2}}\left\langle Z \cdot \phi \phi^{-1}, \bar{Z} \cdot \phi \phi^{-1}\right\rangle=\int_{S^{2}}\langle A, B\rangle$. The degree is defined (up to sign) by $\operatorname{deg} \Phi=\Phi^{*} \omega\left[S^{2}\right] \in \mathbb{Z}$, where $\omega$ is a 2-form representing a generator of $H^{2} \Omega G l_{n} \mathbb{C} \cong \mathbb{Z}$. Now, we may take $\omega$ as $\omega(f, g)=\int_{S^{1}}\left\langle f^{\prime}, g\right\rangle$, where $f, g: S^{1} \rightarrow \mathfrak{g l}_{n} \mathbb{C}$, since this restricts to the well-known generator of $H^{2} \Omega U_{n}$ (see [PS]) and the restriction map is an 
isomorphism on $H^{2}$. Hence we have

$$
\begin{aligned}
\operatorname{deg} \Phi & =\int_{S^{2}} \omega\left(D \Phi(Z) \Phi^{-1}, D \Phi(\bar{Z}) \Phi^{-1}\right)=\int_{S^{2}} \omega\left(Z \cdot \Phi \Phi^{-1}, \bar{Z} \cdot \Phi \Phi^{-1}\right) \\
& =\int_{S^{2}} \int_{S^{1}}\left\langle\left(Z \cdot \Phi \Phi^{-1}\right)^{\prime}, \bar{Z} \cdot \Phi \Phi^{-1}\right\rangle \\
& =\frac{1}{4} \int_{S^{2}} \int_{S^{1}}\left\langle\left(\left(1-\lambda^{-1}\right) A\right)^{\prime},(1-\lambda) B\right\rangle \\
& =(\text { constant }) \times \int_{S^{2}}\langle A, B\rangle \\
& =(\text { constant }) \times E(\phi) .
\end{aligned}
$$

Versions of some of the results in this section have appeared in [Po, $\mathrm{Uh}, \mathrm{Va}$, ZM, ZS].

\section{DRESSING PSEUDO-ACTIONS}

Let $G$ be a group, and let $G_{1}, G_{2}$ be two subgroups with $G_{1} \cap G_{2}=\{I\}$. Then for any $g \in G_{1} G_{2}$ there is a unique factorization $g=g_{1} g_{2}$. With respect to the choice of $G_{1}, G_{2}$, the dressing pseudo-action of $G$ on itself is a certain map $d: E \rightarrow G$, where $E$ is the subset $E=\left\{(g, h) \mid h g h^{-1} \in G_{1} G_{2}\right\}$ of $G \times G$. It is defined by the formula

$$
d(g, h)=g \cdot h=\left(h g h^{-1}\right)_{1}^{-1} h=\left(h g h^{-1}\right)_{2} h g^{-1} .
$$

It may be checked that $g \cdot\left(g^{\prime} \cdot h\right)=\left(g g^{\prime}\right) \cdot h$ whenever $\left(g^{\prime}, h\right),\left(g, g^{\prime}\right.$. $h),\left(g g^{\prime}, h\right) \in E$, so that when $E=G \times G$ we have a genuine action. In particular this is true when $G=G_{1} G_{2}$. The action thus obtained of $G$ on itself preserves the subgroup $G_{1}$, on which it is given explicitly by

$$
g \cdot h=\left(h g h^{-1}\right)_{1}^{-1} h=\left(g h^{-1}\right)_{1}^{-1} \quad\left(h \in G_{1}\right) .
$$

It is essentially the natural action of $G$ on $G / G_{2} \cong G_{1}$.

We shall apply this to the group $G=\Lambda G l_{n} \mathbb{C}$. Let $\Lambda_{1}, \Lambda_{2}$ be subgroups of $\Lambda G l_{n} \mathbb{C}$ with $\Lambda_{1} \cap \Lambda_{2}=\{I\}$, such that the following additional properties hold:

(I) (Extension property) There exist open subsets $U_{1}, U_{2}$ of $S^{2}$ such that $U_{1} \cup U_{2}=S^{2}, U_{1} \cap U_{2} \supseteq S^{1}$, and each $\gamma \in \Lambda_{i}(i=1,2)$ is the restriction of a holomorphic map $U_{i} \rightarrow G l_{n} \mathbb{C}$.

(II) (Normalization property) $\Lambda_{1} \subseteq \Omega G l_{n} \mathbb{C}$.

The motivation for these properties is provided by the "Riemann-Hilbert factorization problem" (see [Ro]). Roughly speaking, this problem asks for a factorization of a map $f: S^{1} \rightarrow G l_{n} \mathbb{C}$ as $f=f_{1} f_{2}$, where $f_{i}$ extends to a holomorphic map on $U_{i}(i=1,2)$. A factorization of this type, which we shall describe in $\S 3$, was used in 1909 by Birkhoff to study singular points of differential equations of the form $d v / d z=A(z) v(z)$ (see 8.2 of [PS]). He found that this equation could be brought to a simple form by making a transformation $A \mapsto Y A$. A more recent application of the method was to the 
study of the KdV equation and related equations (cf. [ZS, Wi]). The philosophy here was different, in that the aim was to find explicit solutions of the equation. By starting with a simple, or "bare" solution, a large class of solutions was obtained by applying "dressing transformations". Our presentation here of the method for the harmonic map equation was inspired by [ZM].

We shall show now how conditions (I) and (II) imply that the dressing pseudoaction on $\Lambda G l_{n} \mathbb{C}$ actually preserves extended solutions.

Lemma 2.1. Let $g \in \Lambda G l_{n} \mathbb{C}$ and let $\Phi: D \rightarrow \Lambda G l_{n} \mathbb{C}$ be a map such that $\widetilde{\Phi}(z)=$ $g \cdot \Phi(z)$ is defined for all $z \in D$. Let $X$ be a vector field on an open subset of $D$, and write $X \cdot \Phi \Phi^{-1}=C$. Then $X \cdot \widetilde{\Phi} \widetilde{\Phi}^{-1}=\widetilde{C}$, where $\widetilde{C}$ is given in terms of $C$ by

$$
\widetilde{C}=\Theta_{1}-1 C \Theta_{1}+X \cdot \Theta_{1}-1 \Theta_{1}=X \cdot \Theta_{2} \Theta_{2}^{-1}+\Theta_{2} C \Theta_{2}^{-1},
$$

where $\Theta=\Phi g \Phi^{-1}$.

Proof. This is a routine verification using the product rule.

Lemma 2.2. Let $C, \widetilde{C}$ be as in Lemma 2.1. If, for a fixed $z \in D$, the matrixvalued function $C(z): S^{1} \rightarrow \mathfrak{g l}_{n} \mathbb{C}$ extends meromorphically to some open set $U$ containing $S^{1}=\{\lambda|| \lambda \mid=1\}$, then so does $\widetilde{C}(z)$. For each $\lambda \in U, C(z)$ has a pole of order $k$ at $\lambda$ if and only if $\widetilde{C}(z)$ does.

Proof. We shall show first that $\widetilde{C}$ extends to $U \cap U_{1}$. By definition, $\Theta_{1}$ extends to a holomorphic function on $U_{1}$, so the first expression for $\widetilde{C}$ in 2.1 (with $X=Z$ ) shows that $\widetilde{C}$ extends to $U \cap U_{1}$. If now $C$ has a pole of order $k$ at $\lambda \in U \cap U_{1}$, then (a) $\Theta_{1}-1 Z \cdot \Theta_{1}$ is holomorphic at $\lambda$, and (b) $\Theta_{1}-1 C \Theta_{1}$ has a pole of order $k$ at $\lambda$. Hence $\widetilde{C}$ has a pole of order $k$ at $\lambda$. A similar argument deals with $U \cap U_{2}$, using the second expression for $\widetilde{C}$ in 2.1 .

Theorem 2.3. Let $g \in \Lambda G l_{n} \mathbb{C}$ and let $\Phi: D \rightarrow \Omega G l_{n} \mathbb{C}$ be an extended solution such that $\widetilde{\Phi}(z)=g \cdot \Phi(z)$ is defined for all $z$ in some open subset $\widetilde{D}$ of $D$. Then $\widetilde{\Phi}: \widetilde{D} \rightarrow \Omega G l_{n} \mathbb{C}$ is an extended solution. Moreover, if $\Phi$ is based and $g \in \Lambda_{2}$, then $\widetilde{\Phi}$ is based.

Proof. We have $C=Z \cdot \Phi \Phi^{-1}=\frac{1}{2}(1-1 / \lambda) A$, which extends meromorphically to the whole of $S^{2}$, having a simple pole at $\lambda=0$ and no other poles. Hence, by 2.1 and $2.2, \widetilde{C}=(1 / \lambda) S+T$ for some $S, T: \widetilde{D} \rightarrow \mathfrak{g l}_{n} \mathbb{C}$. Now the normalization condition (II) above implies that $\Theta_{1}(z)(1)=I$ for all $z$. Hence, by the first formula for $\widetilde{C}$ in 2.1 , we have $\widetilde{C}(z)(1)=C(z)(1)=0$ for all $z$. So $S=-T$ and $Z \cdot \widetilde{\Phi} \widetilde{\Phi}^{-1}$ is of the required form. A similar argument applies to $\bar{Z} \cdot \widetilde{\Phi} \widetilde{\Phi}^{-1}$. The statement concerning basepoints is immediate.

From the point of view of harmonic maps, it is of interest to know to what extent the pseudo-action preserves real extended solutions. 
Proposition 2.4. If $g$ and $\Phi$ in Theorem 2.3 are real, and if $\Phi$ takes values in $\Lambda_{1}$, then $g \cdot \Phi$ is also real.

Proof. It suffices to show that if $g \in \Omega U_{n}, h \in \Omega U_{n} \cap \Lambda_{1}$, and $h g h^{-1} \in \Lambda_{1} \Lambda_{2}$, then $g \cdot h \in \Omega U_{n}$. Let $k=h g h^{-1}$, so that $g \cdot h=k_{1}^{-1} h=k_{2} h g^{-1}$. Then since $g, h$ are real we have $(g \cdot h)(\lambda)^{*}=g(\lambda) h(\lambda)^{-1} k_{2}(\lambda)^{*}$ and $(g \cdot h)(\lambda)^{-1}=$ $g(\lambda) h(\lambda)^{-1} k_{2}(\lambda)^{-1}$. Now, $g \cdot h \in \Lambda_{1}$ (as $\left.h \in \Lambda_{1}\right)$, so we have just obtained two decompositions of $g(\lambda) h(\lambda)^{-1}$ of the form $f_{1} f_{2}$ with $f_{1} \in \Lambda_{1}, f_{2} \in \Lambda_{2}$. As $\Lambda_{1} \cap \Lambda_{2}=\{I\}$, these decompositions must coincide. Hence $(g \cdot h)(\lambda)^{*}=$ $(g \cdot h)(\lambda)^{-1}$, so $g \cdot h\left(\right.$ and $\left.k_{2}\right)$ are real.

\section{THE BIRKHOFF PSEUdO-ACTION}

We recall first the well-known "Birkhoff decomposition" of $\Lambda G l_{n} \mathbb{C}$ (see 8.1.2 of [PS]). Let $\Delta$ be the subgroup of $\Lambda G l_{n} \mathbb{C}$ consisting of homomorphisms from $S^{1}$ to the subgroup of diagonal matrices in $G l_{n} \mathbb{C}$. Thus, $\gamma \in \Delta$ if and only if $\gamma(\lambda)$ is a diagonal matrix with diagonal terms $\lambda^{a_{1}}, \ldots, \lambda^{a_{n}}$ for some integers $a_{1}, \ldots, a_{n}$. Let

$$
\begin{array}{r}
\Lambda_{+}=\left\{\gamma \in \Lambda G l_{n} \mathbb{C} \mid \gamma\right. \text { extends continuously } \\
\text { to a holomorphic map on }|\lambda|<1\}, \\
\Lambda_{-}=\left\{\gamma \in \Lambda G l_{n} \mathbb{C} \mid \gamma\right. \text { extends continuously } \\
\text { to a holomorphic map on }|\lambda|>1\},
\end{array}
$$

and

$$
\Lambda_{-}^{*}=\left\{\gamma \in \Lambda_{-} \mid \gamma(1)=1\right\} .
$$

(By "extends continuously to a holomorphic map on $|\lambda|<1$ " we mean "extends to a continuous map on $|\lambda| \leq 1$, which is holomorphic on $|\lambda|<1$ ".)

Theorem 3.1. $\Lambda G l_{n} \mathbb{C}=\Lambda_{-} \Delta \Lambda_{+}$. Moreover, $\Lambda_{-} \Lambda_{+}$is a dense open subspace of the identity component of $\Lambda G l_{n} \mathbb{C}$, and the multiplication map $\Lambda_{-}^{*} \times \Lambda_{+} \rightarrow$ $\Lambda G l_{n} \mathbb{C}$ is a diffeomorphism onto this subspace.

If $\gamma \in \Lambda G l_{n} \mathbb{C}$ is considered as a clutching function for a holomorphic vector bundle on $S^{2}$, then the diagonal term in the factorization of $\gamma$ given by this theorem determines the isomorphism class of the bundle (see 8.2 of [PS]). This is a well-known observation of Grothendieck.

Definition 3.2. The Birkhoff pseudo-action of $\Lambda G l_{n} \mathbb{C}$ on itself is defined by $g \cdot h=\left(h g h^{-1}\right)_{1}^{-1} h=\left(h g h^{-1}\right)_{2} h g^{-1}$, where $g, h \in \Lambda G l_{n} \mathbb{C}$, and where $\Lambda_{1}=$ $\Lambda_{-}^{*}, \Lambda_{2}=\Lambda_{+}$.

Unfortunately this choice of $\Lambda_{1}, \Lambda_{2}$ does not satisfy the extension property (I) of $\S 2$, since the obvious candidates for $U_{1}, U_{2}$ are open neighbodurhoods of the two hemispheres, but a map $\gamma \in \Lambda_{i}$ does not necessarily extend to a holomorphic map of $U_{i}$. However, it is easy to extend Lemma 2.2 to cover this 
situation (the proof of 2.2 shows that $\widetilde{C}$ extends to $U \cap\left(S^{2}-S^{1}\right)$, but since $\widetilde{C}$ is already defined on $S^{1}=\{\lambda|| \lambda \mid=1\}$, it is defined on $U \cap S^{1}$ as well). Hence Theorem 2.3 and Proposition 2.4 are also valid for the Birkhoff pseudo-action.

The extent to which this induces an action of $\Lambda G l_{n} \mathbb{C}$ on extended solutions, as opposed to just a pseudo-action, is governed by Theorem 2.3 (or, rather, the generalization of Theorem 2.3 just mentioned). In general, it seems to be difficult to tell whether the image of $\Phi g \Phi^{-1}$ lies in $\Lambda_{1} \Lambda_{2}$. However, if $g \in \Lambda_{1} \Lambda_{2}$, then $\Phi g \Phi^{-1}(*)=g \in \Lambda_{1} \Lambda_{2}$, so $\left(\Phi g \Phi^{-1}\right)^{-1}\left(\Lambda_{1} \Lambda_{2}\right)$ is a nonempty open subset $\widetilde{D}$ of $D$, and we are in the situation of Theorem 2.3. Moreover, the complement of $\widetilde{D}$ is small in the sense that it has empty interior: this follows from the fact that an extended solution is necessarily real analytic, and that the complement of $\Lambda_{1} \Lambda_{2}$ is a real analytic submanifold of $\Lambda G l_{n} \mathbb{C}$. In addition, we have the following important fact:

Proposition 3.3. Let $\Phi: S^{2} \rightarrow \Omega G l_{n} \mathbb{C}$ be an extended solution. Let $g \in \Lambda G l_{n} \mathbb{C}$, and assume that the extended solution $g \cdot \Phi$ is defined on the whole of $S^{2}$. Then $\operatorname{deg} g \cdot \Phi=\operatorname{deg} \Phi$.

Proof. The hypotheses imply that $\Theta_{1}-1=\left(\Phi g \Phi^{-1}\right)_{1}^{-1}$ is defined on the whole of $S^{2}$, so $\operatorname{deg} g \cdot \Phi=\operatorname{deg} \Theta_{1}-1+\operatorname{deg} \Phi$. Note that $\pi_{2} \Lambda_{-}^{*}=0$; in fact $\Lambda_{-}^{*}$ is contractible, via the obvious homotopy which shrinks the domain from the unit disc to the point 1 . Hence $\operatorname{deg} \Theta_{1}=0$ and so $\operatorname{deg} g \cdot \Phi=\operatorname{deg} \Phi$.

Hence (cf. Proposition 1.3), the Birkhoff pseudo-action of $\Omega G l_{n} \mathbb{C}$ on harmonic maps $S^{2} \rightarrow G l_{n} \mathbb{C}$ preserves energy when it is defined.

It should be emphasized that if $\Phi$ is real, there is no suggestion that $g \cdot \Phi$ is real. In fact:

Proposition 3.4. If $\Phi$ and $g \cdot \Phi$ are both real, then they are equal.

Proof. We have $g \cdot \Phi=\left(\Phi g \Phi^{-1}\right)_{1}^{-1} \Phi$, so $\left(\Phi g \Phi^{-1}\right)_{1}^{-1}$ is also real, i.e., $\Omega U_{n}$ valued. But it is well known that $\Lambda_{1} \cap \Omega U_{n}=\Lambda_{-}^{*} \cap \Omega U_{n}$ consists only of the identity loop (see 8.1.1 of [PS]), so $\left(\Phi g \Phi^{-1}\right)_{1}^{-1}=I$ and $\Phi=g \cdot \Phi$.

Thus, the Birkhoff pseudo-action induces the trivial action amongst real based extended solutions (and hence amongst harmonic maps $D \rightarrow U_{n}$ ). For this reason, we shall need to consider more general pseudo-actions.

\section{GeNeralized BirkHoff PSEUdo-ACTIONS}

The Birkhoff action of the previous section may be generalized by replacing the equator $\{\lambda|| \lambda \mid=1\}$ by a finite collection of disjoint circles $C_{1}, \ldots, C_{k}$. A version of this construction has been considered by Avan and Bellon in [AB] and by Ohnita in unpublished work. We shall assume that each circle $C_{i}$ is oriented, so that it has an interior $I_{i}$ and an exterior $E_{i}$, and furthermore that

(i) the interiors $I_{1}, \ldots, I_{k}$ of the circles are mutually disjoint, and

(ii) the exteriors $E_{1}, \ldots, E_{k}$ all contain the equator.

Let $I=I_{1} \cup \cdots \cup I_{k}, E=E_{1} \cap \cdots \cap E_{k}$. 
Theorem 4.1. Let $g=\left(g_{1}, \ldots, g_{k}\right): C_{1} \cup \cdots \cup C_{k} \rightarrow G l_{n} \mathbb{C}$ be a smooth map. Then there exist smooth maps $g^{E}, g^{I}: C_{1} \cup \cdots \cup C_{k} \rightarrow G l_{n} \mathbb{C}$ and a diagonal homomorphism $\delta: C_{1} \rightarrow G l_{n} \mathbb{C}$ such that (1) $g=g^{E}(\delta, 1, \ldots, 1) g^{I}$, and (2) $g^{E}, g^{I}$ extend continuously to holomorphic maps $G^{E}, G^{I}$ of $E, I$ respectively.

The proof will be given later. To say that $\delta$ is a diagonal homomorphism of the circle $C_{1}=\{\lambda|| \lambda-a \mid=r\}$ into $G l_{n} \mathbb{C}$ means that $\delta(\lambda)$ is a diagonal matrix, whose diagonal terms are powers of $(\lambda-a) / r$.

The role of the loop space in $\S 3$ is now played by the product of loop spaces

$$
\Lambda^{1, \ldots, k} G l_{n} \mathbb{C}=\Lambda^{1} G l_{n} \mathbb{C} \times \cdots \times \Lambda^{k} G l_{n} \mathbb{C},
$$

where $\Lambda^{i} G l_{n} \mathbb{C}=\left\{\gamma: C_{i} \rightarrow G l_{n} \mathbb{C} \mid \gamma\right.$ smooth $\} \cong \Lambda G l_{n} \mathbb{C}$. We have subgroups $\Lambda_{E}, \Lambda_{I}$ consisting of those elements of $\Lambda^{1, \ldots, k} G l_{n} \mathbb{C}$ which extend continuously to holomorphic maps of $E, I$ respectively, and we define

$$
\Lambda_{E}^{*}=\left\{\gamma \in \Lambda_{E} \mid \gamma^{E}(1)=1\right\} \text {. }
$$

Then as in Theorem 3.1 we have a decomposition $\Lambda^{1, \ldots, k} G l_{n} \mathbb{C}=\Lambda_{E} \Delta \Lambda_{I}$, and the multiplication map $\Lambda_{E}^{*} \times \Lambda_{I} \rightarrow \Lambda^{1, \ldots, k} G l_{n} \mathbb{C}$ is a diffeomorphism onto the dense open subset $\Lambda_{E} \Lambda_{I}$ of a component of $\Lambda^{1, \ldots, k} G l_{n} \mathbb{C}$.

Definition 4.2. The generalized Birkhoff pseudo-action of $\Lambda^{1, \ldots, k} G l_{n} \mathbb{C}$ on itself is defined by $g \cdot h=\left(h g h^{-1}\right)_{E}^{-1} h=\left(h g h^{-1}\right)_{I} h g^{-1}$, where $g, h \in \Lambda^{1, \ldots, k} G l_{n} \mathbb{C}$, and where we write $f=f_{E} f_{I}$ for the unique factorization of $f \in \Lambda_{E}^{*} \Lambda_{I}$.

Theorem 4.1 and Definition 4.2 are natural generalizations of 3.1 and 3.2. The following generalization of Theorem 2.3 is then obtained.

Theorem 4.3. Let $C_{1}, \ldots, C_{k}$ be circles satisfying (i) and (ii) above. Let $g \in$ $\Lambda^{1, \ldots, k} G l_{n} \mathbb{C}$, and let $\Phi: D \rightarrow \Omega G l_{n} \mathbb{C}$ be an extended solution such that $\Phi(z)(\lambda)$ is defined for all $\lambda \in C_{1} \cup \cdots \cup C_{k}$. If $\widetilde{\Phi}(z)=g \cdot \Phi(z)$ is defined for all $z$ in some open subset $\widetilde{D}$ of $D$, then $\widetilde{\Phi}: \widetilde{D} \rightarrow \Omega G l_{n} \mathbb{C}$ is an extended solution. Moreover, if $\Phi$ is based, and $g \in \Lambda_{I}$, then $\widetilde{\Phi}$ is based.

Proof. This follows the proof of Theorem 2.3 and its generalization described in $\S 3$.

A based extended solution is defined for all $\lambda \in \mathbb{C}^{*}$. If the circles are such that

(iii) $0, \infty \in I$

then any based extended solution is defined on $C_{1} \cup \cdots \cup C_{k}$. Hence we have:

Corollary 4.4. If the circles $C_{1}, \ldots, C_{k}$ satisfy (i), (ii), and (iii), then we have a pseudo-action of $\Lambda_{I}$ on based extended solutions. 
As with the Birkhoff pseudo-action, if $g \in \Lambda_{E} \Lambda_{I}$, then $\left(\Phi g \Phi^{-1}\right)^{-1}\left(\Lambda_{E} \Lambda_{I}\right)$ is a nonempty open subset $\widetilde{D}$ of $D$, so the situation of Theorem 4.3 is not vaccuous.

Proposition 3.3 extends directly to generalized Birkhoff pseudo-actions:

Proposition 4.5. Let $\Phi: S^{2} \rightarrow \Omega G l_{n} \mathbb{C}$ be an extended solution. Let $g \in$ $\Lambda^{1, \ldots, k} G l_{n} \mathbb{C}$, and assume that the extended solution $g \cdot \Phi$ is defined on the whole of $S^{2}$. Then $\operatorname{deg} g \cdot \Phi=\operatorname{deg} \Phi$.

Proof. The proof of 3.3 must be modified as $\Lambda_{E}^{*}$ is not contractible in general. However, $\Lambda_{I}$ has $G l_{n} \mathbb{C} \times \cdots \times G l_{n} \mathbb{C}$ ( $k$ factors) as a deformation retract, hence $\pi_{2} \Lambda_{I}=0$ and so we may apply the argument of 3.3 to the formula $g \cdot \Phi=\left(\Phi g \Phi^{-1}\right)_{I}^{-1} \Phi g^{-1}$.

The important new feature is that Proposition 3.4 does not necessarily carry over to generalized Birkhoff pseudo-actions: we have potentially interesting actions on real extended solutions and hence on harmonic maps $D \rightarrow U_{n}$. To explain this, we need some remarks on the appropriate reality conditions. Recall that an extended solution $\Phi$ is real if and only if $\Phi(z)(\lambda)^{*}=\Phi(z)(\lambda)^{-1}$, for all $z \in D$ and for all $\lambda$ with $|\lambda|=1$. If $\Phi$ is based (and hence defined for all $\left.\lambda \in \mathbb{C}^{*}\right)$, this reality condition is equivalent to $\Phi(z)(1 / \bar{\lambda})^{*}=\Phi(z)(\lambda)^{-1}$, for all $z \in D$ and for all $\lambda \in \mathbb{C}^{*}$. We shall deal only with based extended solutions from now on in order to ensure that they are defined on the circles $C_{1}, \ldots, C_{k}$. We shall say that a $k$-tuple $g=\left(g_{1}, \ldots, g_{k}\right) \in \Lambda^{1, \ldots, k} G l_{n} \mathbb{C}$ is real if and only if it satisfies the condition $g(1 / \bar{\lambda})^{*}=g(\lambda)^{-1}$ for all $\lambda \in C_{1} \cup \cdots \cup C_{k}$. It is implicitly assumed that the set $C_{1} \cup \cdots \cup C_{k}$ is preserved by the transformation $\lambda \mapsto 1 / \bar{\lambda}$. If $g=\left(g_{1}, \ldots, g_{k}\right) \in \Lambda^{1, \ldots, k} G l_{n} \mathbb{C}$ is real, and if $\Phi$ is a based real extended solution which takes values in $\Lambda_{E}^{*}$, then $g \cdot \Phi$ is also real by the argument of Proposition 2.4. In the next section we shall examine one such action.

Proof of Theorem 4.1.

Step 1 (the case $k=1$ ). This is essentially the Birkhoff decomposition of $\S 3$, except that the equator has been replaced by any circle $C$. It is well known that there is a linear fractional transformation $z \mapsto f(z)$ which takes the equator to $C$ and the hemisphere $\{\lambda|| \lambda \mid<1\}$ to the interior of $C$. Given a smooth map $g: C \rightarrow G l_{n} \mathbb{C}$, we have a Birkhoff decomposition $g f=g_{-} \delta g_{+}$. It is easily verified that the required factorization of $g$ is $g=\left(g_{-} f^{-1}\right)\left(\delta f^{-1}\right)\left(g_{+} f^{-1}\right)$.

Step 2 (the case $k=2$ ). Let $g=\left(g_{1}, g_{2}\right): C_{1} \cup C_{2} \rightarrow G l_{n} \mathbb{C}$. We may consider $\left(1, g_{2}\right)$ as defining a holomorphic vector bundle on $E_{1}$ (see 8.10 of [PS]). Such a bundle is necessarily holomorphically trivial, as $E_{1}$ is a Stein manifold, so (by Step 1) we have a factorization $g_{2}=g_{2}^{E} g_{2}^{I_{2}}$ (with no "diagonal term"). Here, $g_{2}^{E}, g_{2}^{I_{2}}$ extend to holomorphic maps $G_{2}^{E}, G_{2}^{I_{2}}$ of $E, I_{2}$ respectively. So 


$$
\begin{aligned}
\left(g_{1}, g_{2}\right) & =\left(g_{1}, 1\right)\left(1, g_{2}\right)=\left(g_{1}, 1\right)\left(1, g_{2}^{E}\right)\left(1, g_{2}^{I_{2}}\right) \\
& =\left(g_{2}^{E}, g_{2}^{E}\right)\left(\left(g_{2}^{E}\right)^{-1} g_{1}, 1\right)\left(1, g_{2}^{I_{2}}\right) .
\end{aligned}
$$

We use the obvious convention here that $\left(g_{2}^{E}, 1\right)$ means $\left(\left.G_{2}^{E}\right|_{C_{1}}, 1\right)$, etc. Let us now write $h=\left(g_{2}^{E}\right)^{-1} g_{1}$. Performing a factorization of $h$ with respect to the circle $C_{1}$ (by Step 1 again), we obtain $h=h^{E_{1}} \delta h^{I_{1}}$. So $(h, 1)=$ $\left(h^{E_{1}}, h^{E_{1}}\right)(\delta, 1)\left(1,\left(h^{E_{1}}\right)^{-1}\right)\left(h^{I_{1}}, 1\right)$. Hence we have

$$
\left(g_{1}, g_{2}\right)=\left(g_{2}^{E} h^{E_{1}}, g_{2}^{E} h^{E_{1}}\right)(\delta, 1)\left(1,\left(h^{E_{1}}\right)^{-1}\right)\left(h^{I_{1}}, g_{2}^{I_{2}}\right)
$$

which is of the required form $g^{E}(\delta, 1) g^{I}$, with $G^{E}=G_{2}^{E} H^{E_{1}}$ and $G^{I}=$ $\left(H^{I_{1}},\left(H^{E_{1}}\right)^{-1} G_{2}^{I_{2}}\right)$.

Step 3 (the general case $k \geq 2$ ). The proof is by induction on $k$. Step 2 begins the induction. Assume that the theorem is true for some integer $k(\geq 2)$. If $g=\left(g_{1}, \ldots, g_{k+1}\right): C_{1} \cup \cdots \cup C_{k} \rightarrow G l_{n} \mathbb{C}$, we may write

$$
g=\left(g_{1}, \ldots, g_{k}, 1\right)\left(1, \ldots, 1, g_{k+1}\right) .
$$

As in Step 2 we obtain a factorization $g_{k+1}=g_{k+1}^{E} g_{k+1}^{I_{k+1}}$. Hence

$$
g=\left(g_{k+1}^{E}, \ldots, g_{k+1}^{E}\right)\left(\left(g_{k+1}^{E}\right)^{-1} g_{1}, \ldots,\left(g_{k+1}^{E}\right)^{-1} g_{k}, 1\right)\left(1, \ldots, 1, g_{k+1}^{I_{k+1}}\right) .
$$

The first and third factors here are of the correct form. By the inductive hypothesis, the middle term may be written as $p(\delta, 1, \ldots, 1) q$, where $p, q$ extend to $E_{1} \cap \cdots \cap E_{k}, I_{1} \cup \cdots \cup I_{k}$ respectively. But then $p$ certainly extends to $E$, and $q$ extends to $I$ by defining it to be 1 on $I_{k+1}$. So $g$ may be written in the correct form, and the theorem follows by induction.

\section{THE POLAR PSEUdo-ACTION OF UHLENBECK}

Let $C(r)$ denote the circle $\{\lambda|| \lambda \mid=r\}$ in $\mathbb{C} \cup \infty=S^{2}$, and let $\Lambda(r)=$ $\left\{\gamma: C(r) \rightarrow G l_{n} \mathbb{C} \mid \gamma\right.$ smooth $\}$. We shall apply the theory of $\S 4$ to the choice of circles $C_{1}=C(\varepsilon), C_{2}=C(1 / \varepsilon)$, where $0<\varepsilon<1$. These circles will be oriented so that the interiors $I(\varepsilon), I(1 / \varepsilon)$ are neighborhoods of $0, \infty$ (the north and south poles), respectively. Let

$$
\Lambda(\varepsilon, 1 / \varepsilon)=\Lambda(\varepsilon) \times \Lambda(1 / \varepsilon) \cong \Lambda G l_{n} \mathbb{C} \times \Lambda G l_{n} \mathbb{C} .
$$

The subgroups $\Lambda_{E}, \Lambda_{E}^{*}, \Lambda_{I}$ of $\Lambda(\varepsilon, 1 / \varepsilon)$ are defined as in $\S 4$.

By Corollary 4.4 , we have a pseudo-action of $\Lambda_{I}$ on extended solutions. By the extension of Proposition 2.4, this restricts to a pseudo-action of real elements of $\Lambda_{I}$ on real extended solutions. By Proposition 4.5, the pseudo-action preserves the degree of extended solutions on $S^{2}$ (or the energy of harmonic maps of $S^{2}$ ). This pseudo-action will be called the polar pseudo-action . 
Example 5.1. Let $\Phi$ be an extended solution. Let $g(\lambda)=A \in G l_{n} \mathbb{C}$ be a constant loop. Then certainly $g \in \Lambda_{I}$, so we know by Corollary 4.4 that $g \cdot \Phi$ is an extended solution on a nonempty open subset of $S^{2}$. In fact we have

$$
\begin{aligned}
g \cdot \Phi & =\left(\Phi g \Phi^{-1}\right)_{E}^{-1} \Phi=\left(g \Phi^{-1}\right)_{E}^{-1} \quad \text { as } \Phi(z) \in \Lambda_{E}^{*} \\
& =\left(A \Phi^{-1} A^{-1} A\right)_{E}^{-1}=A_{E}^{-1} A \Phi A^{-1} \text { as } A \Phi(z) A^{-1} \in \Lambda_{E}^{*} \\
& =A \Phi A^{-1} .
\end{aligned}
$$

Thus $g \cdot \Phi$ is actually defined on the whole of $S^{2}$. For Example, let $\Phi=$ $\pi_{f}^{\perp}+\lambda \pi_{f}$ as in example 1.2, where $f: S^{2} \rightarrow \mathrm{Gr}_{k}\left(\mathbb{C}^{n}\right)$ is a holomorphic map. Assume $g$ is real, i.e., $A \in U_{n}$. Then $g \cdot \Phi=\pi_{A f}^{\perp}+\lambda \pi_{A f}$, which is the extended solution associated to the holomorphic map $A f$. So we see that on real extended solutions the polar pseudo-action (in contrast to the Birkhoff pseudo-action) is definitely not trivial.

The polar pseudo-action is closely related to an operation introduced by Uhlenbeck in [Uh], as we shall now explain. Since we are considering only $g \in \Lambda_{I}$ and $\Phi(z) \in \Lambda_{E}^{*}$, the formula for the pseudo-action simplifies (as in Example 5.1) to

$$
g \cdot \Phi=\left(g \Phi^{-1}\right)_{E}^{-1}=\left(g \Phi^{-1}\right)_{I} \Phi g^{-1} .
$$

Hence it would be possible to define the pseudo-action by $g \cdot \Phi=h \Phi g^{-1}$, where $h$ is that unique element of $\Lambda_{I}$ (if such an element exists) with the property that $h \Phi g^{-1}$ is $\Lambda_{E}^{*}$-valued. The procedure of [Uh] is essentially this, restricted to "meromorphic" functions. The singularities are now only poles and zeros of a matrix-valued function, and one has the picturesque analytical interpretation that " $h$ introduces singularities on the left, and $g^{-1}$ takes them off again on the right".

We shall now give a useful characterization of the pseudo-action of $\Lambda_{I}$. Since the action of any $g \in \Lambda_{E}$ on an extended solution $\Phi$ is given simply by right multiplication by $g^{-1}$ (as $\left.g \cdot \Phi=\left(\Phi g \Phi^{-1}\right)_{E}^{-1} \Phi=\Phi g^{-1}\right)$, this will allow us to deal with the pseudo-action of any $g \in \Lambda_{E} \Lambda_{I}$. Before stating the characterization, we shall need a simple property of solutions of the linear equations

$$
Z \cdot \Phi \Phi^{-1}=\frac{1}{2}(1-1 / \lambda) A, \quad \bar{Z} \cdot \Phi \Phi^{-1}=\frac{1}{2}(1-\lambda) B .
$$

(This property was hinted at in Remark 1 of $\S 1$.)

Lemma 5.2. Let $A, B: D \rightarrow \mathfrak{g l}_{n} \mathbb{C}$ be smooth functions. Let $U$ be a nonempty open subset of $\mathbb{C}^{*}$. Let $\Phi: D \times U \rightarrow G l_{n} \mathbb{C},(z, \lambda) \mapsto \Phi(z)(\lambda)$ be a smooth function which satisfies the above linear equations. Assume further that $\Phi$ is based, i.e., $\Phi(*)(\lambda)=I$ for all $\lambda \in U$. Then $\Phi$ is the restriction (to $U$ ) of a based extended solution. (Recall from Remark 1 of $\S 1$ that a based extended solution may be considered as defined for all $\lambda \in \mathbb{C}^{*}$.) 
Proof. The proof is contained essentially in the proof of Proposition 1.1, so we sketch it briefly, using the notation of that proposition. First, the linear equations imply that $d \omega_{\lambda}-\frac{1}{2}\left[\omega_{\lambda} \wedge \omega_{\lambda}\right]=0$ as in 1.1 , where $\omega_{\lambda}=(1-1 / \lambda) A d z / 2+$ $(1-\lambda) B d \bar{z} / 2$. Hence, for each $\lambda \in \mathbb{C}^{*}, \omega_{\lambda}=d \Phi_{\lambda} \Phi_{\lambda}^{-1}$ for some function $\Phi_{\lambda}$ with $\Phi_{\lambda}(*)=I$. Since $\Phi_{\lambda}$ is unique, we necessarily have $\Phi_{\lambda}(z)=\Phi(z)(\lambda)$ for all $\lambda \in U$.

Theorem 5.3. Let $\Phi$ be a based extended solution.

(1) Let $\Psi: D \rightarrow \Lambda_{I}$ be such that $\Psi \Phi$ satisfies the linear equations, for $\lambda$ in some nonempty open subset of the Riemann sphere. Let $g=\Psi(*)$ $\left(\in \Lambda_{I}\right)$. Then $g \cdot \Phi$ is defined for all $z \in D$, and is equal to $\Psi \Phi g^{-1}$.

(2) Conversely, let $g \in \Lambda_{I}$, and assume that $g \cdot \Phi$ is defined for all $z \in D$. Then $g=\Psi(*)$ for some function $\Psi: D \rightarrow \Lambda_{I}$ such that $\Psi \Phi$ satisfies the linear equations (on a nonempty open subset of the Riemann sphere).

Proof. (1) Since multiplication on the right does not alter the property of being a solution of the linear equations, the map $\Psi \Phi g^{-1}$ is also a solution. By construction, it is based. Hence by Lemma 5.2, $\Psi \Phi g^{-1}$ extends to a based extended solution, i.e., it is $\Lambda_{E}^{*}$-valued. Now, by the earlier remarks, if $g \cdot \Phi$ exists then it is of the form $\Delta \Phi g^{-1}$, and $\Delta$ is uniquely determined by the properties that (a) $\Delta$ is $\Lambda_{I}$-valued, and (b) $\Delta \Phi g^{-1}$ is $\Lambda_{E}^{*}$-valued. Since $\Delta=\Psi$ satisfies (a) and (b), we are done.

(2) We have $g \cdot \Phi=\Psi \Phi g^{-1}$ for some function $\Psi: D \rightarrow \Lambda_{I}$. Since $g \cdot \Phi$ is based, evaluation at $z=*$ gives $I=\Psi(*) g^{-1}$, i.e., $g=\Psi(*)$. Since $\Psi \Phi g^{-1}$ is an extended solution, $\Psi \Phi$ satisfies the linear equations.

Hence the (rather inaccesible) problem of deciding when $\Psi(*) \cdot \Phi$ is defined has been converted into the (more appealing) problem of deciding when $\Psi \Phi$ satisfies the linear equations.

A natural class of examples is provided by functions $\Psi$ which are "rational" in $\lambda$. The criterion of Theorem 5.3 can be expressed in terms of the residues and singular values of $\Psi$. To make this precise, let $\Psi$ be a $\mathfrak{g l}_{n} \mathbb{C}$-valued rational function on $D$ in the sense that for each $z \in D$, each component of $\Psi(z)$ is a rational function in $\lambda$. We shall call $\Psi$ a $G l_{n} \mathbb{C}$-valued rational function if in addition (a) $\Phi$ has poles at the singular points $b_{1}, \ldots, b_{k}$ of $\Phi^{-1}$, (b) $\Phi$ has singular points at the poles $a_{1}, \ldots, a_{l}$ of $\Phi^{-1}$, where $a_{i} \neq b_{j}$ for all $i, j$, and (c) $\Psi(\lambda) \in G l_{n} \mathbb{C}$ for all $\lambda \neq a_{i}, b_{j}$. (A singular point means a point where the determinant is zero.)

Theorem 5.4. Let $\Phi$ be a based extended solution. Let $\Psi:(z, \lambda) \mapsto \Psi(z)(\lambda)$ be a $G l_{n} \mathbb{C}$-valued rational function in the above sense. Assume that $\Psi(z)(1)=I$ for all $z \in D$. Then $\Psi \Phi$ satisfies the linear equations if and only if the singular points $a_{1}, \ldots, a_{l}$ and the poles $b_{1}, \ldots, b_{k}$ of $\Psi$ are independent of $z$ and the 
following $2 k+2 l$ equations are satisfied:

$$
\begin{aligned}
& \Psi\left(a_{i}\right)\left(Z-\frac{1}{2}\left(1-1 / a_{i}\right) A\right) \operatorname{Res}\left(\Psi^{-1}, a_{i}\right)=0, \\
& \operatorname{Res}\left(\Psi, b_{i}\right)\left(Z-\frac{1}{2}\left(1-1 / b_{i}\right) A\right) \Psi^{-1}\left(b_{i}\right)=0, \\
& \Psi\left(a_{i}\right)\left(\bar{Z}-\frac{1}{2}\left(1-a_{i}\right) B\right) \operatorname{Res}\left(\Psi^{-1}, a_{i}\right)=0, \\
& \quad \operatorname{Res}\left(\Psi, b_{i}\right)\left(\bar{Z}-\frac{1}{2}\left(1-b_{i}\right) B\right) \Psi^{-1}\left(b_{i}\right)=0 \\
& (i=1, \ldots, l, j=1, \ldots, k) .
\end{aligned}
$$

Proof. We have

$$
Z \cdot(\Psi \Phi)(\Psi \Phi)^{-1}=\Psi\left[\frac{1}{2}(1-(1 / \lambda)) A\right] \Psi^{-1}+Z \cdot \Psi \Psi^{-1} .
$$

Since $Z \cdot \Psi \Psi^{-1}=Z-\Psi Z \Psi^{-1}$ (see $\S 1$ and also [Gu2]), this simplifies to $Z-\Psi\left[Z-\frac{1}{2}(1-(1 / \lambda)) A\right] \Psi^{-1}$. Similarly we have $\bar{Z} \cdot(\Psi \Phi)(\Psi \Phi)^{-1}=\bar{Z}-$ $\Psi\left[\bar{Z}-\frac{1}{2}(1-\lambda) B\right] \Psi^{-1}$.

If the poles and singular points of $\Psi$ are independent of $z$, and if the $2 k+2 l$ equations are satisfied, then we see that $Z \cdot(\Psi \Phi)(\Psi \Phi)^{-1}$ is meromorphic in $\lambda$ with a simple pole at 0 and with possible poles at the points $a_{i}, b_{j}$. However, the first $k+l$ equations say precisely that the residues at the points $a_{i}, b_{j}$ are all zero. Hence $Z \cdot(\Psi \Phi)(\Psi \Phi)^{-1}$ is of the form $(1 / \lambda) S+T$. Since $\Psi(z)(1)=I$ for all $z, Z \cdot(\Psi \Phi)(\Psi \Phi)^{-1}$ is zero when $\lambda=1$, so we have $T=-S$. Similarly we find $\bar{Z} \cdot(\Psi \Phi)(\Psi \Phi)^{-1}$ is of the form $\lambda U+V$ with $V=-U$. Hence $\Psi \Phi$ satisfies the linear equations.

Conversely, assume that $\Psi \Phi$ satisfies the linear equations. Then the map $\Psi \Phi(\Psi \Phi)(*)^{-1}$ is a based extended solution. Since the poles of $\Phi$ and of $\Psi \Phi(*)^{-1}$ are independent of $z$, so are those of $\Psi$. The singular points are independent of $z$ for a similar reason. The $2 k+2 l$ equations now follow from the calculation in the first paragraph above.

In the real case one has $k=l$ and $b_{i}=1 / \bar{a}_{i}$ (after reordering the indices if necessary), and

$$
\operatorname{Res}\left(\Psi, b_{i}\right)=-b_{i}^{2} \operatorname{Res}\left(\Psi^{-1}, 1 / \bar{b}_{i}\right)^{*}, \quad \Psi\left(1 / \bar{b}_{i}\right)=\Psi^{-1}\left(b_{i}\right)^{*} .
$$

Hence the $4 k$ equations reduce to the following system of $2 k$ equations:

$$
\begin{gathered}
\Psi\left(1 / \bar{b}_{i}\right)\left(Z-\frac{1}{2}\left(1-\bar{b}_{i}\right) A\right) \operatorname{Res}\left(\Psi^{-1}, 1 / \bar{b}_{i}\right)=0, \\
\operatorname{Res}\left(\Psi, b_{i}\right)\left(Z-\frac{1}{2}\left(1-1 / b_{i}\right) A\right) \Psi^{-1}\left(b_{i}\right)=0 .
\end{gathered}
$$

As an example, take $\Psi$ of the form

$$
\Psi(z)(\lambda)=(I-P(z))+\frac{(\lambda-a)(1-b)}{(\lambda-b)(1-a)} P(z)
$$

(which we shall abbreviate to $\Psi=(I-P)+\xi P)$, where $a, b$ are complex numbers and $P(z)$ is a projection matrix for all $z$ (i.e., $P^{2}=P$ ). If $a, b \neq$ $0, \infty$, then $\Psi(z) \in \Lambda_{I}$ and $\Psi(z)(1)=I$. Note that $\Psi^{-1}=(I-P)+\xi^{-1} P$. 
The condition that $\Psi \Phi$ be an extended solution is therefore equivalent to

$$
\begin{gathered}
(I-P)\left(Z-\frac{1}{2}(1-1 / a) A\right) P=0, \\
P\left(Z-\frac{1}{2}(1-1 / b) A\right)(I-P)=0, \\
(I-P)\left(\bar{Z}-\frac{1}{2}(1-a) B\right) P=0, \\
P\left(\bar{Z}-\frac{1}{2}(1-b) B\right)(I-P)=0 .
\end{gathered}
$$

(These equations arise also in the context of the "Riemann problem with zeros" in $\S 5$ of [ZM], and in the context of Bäcklund transformations in $\S 6$ of [Uh].)

Now, $\Psi=(I-P)+\xi P$ is real if and only if $P^{*}=P$ and $a=1 / \bar{b}$. In this case we may write $P^{\perp}$ for $I-P$, and $\Psi \Phi$ satisfies the linear equations if and only if

$$
P^{\perp}\left(Z-\frac{1}{2}(1-\bar{b}) A\right) P=0, \quad P\left(Z-\frac{1}{2}(1-1 / b) A\right) P^{\perp}=0 .
$$

Example 5.5 (cf. Theorem 5.3 of [Uh]). Let $\Phi$ be a real based extended solution. Let $g(\lambda)=\pi_{V}^{\perp}+\xi(\lambda) \pi_{V}$, where $\xi(\lambda)=((\lambda-a) /(\lambda-1 / \bar{a}))((1-1 / \bar{a}) /(1-a))$ as above. Let $\Psi(\lambda)=\pi_{\Phi(a) V}^{\perp}+\xi(\lambda) \pi_{\Phi(a) V}$. Thus, $\Psi(*)(\lambda)=g(\lambda)$. We can deduce from Theorem 5.3 that $g \cdot \Phi=\Psi \Phi g^{-1}$ if we show that $\Psi \Phi$ satisfies the linear equations

$$
\begin{gathered}
\pi_{\Phi(a) V}^{\perp}\left(Z-\frac{1}{2}(1-1 / a) A\right) \pi_{\Phi(a) V}=0 \\
\pi_{\Phi(a) V}\left(Z-\frac{1}{2}(1-\bar{a}) A\right) \pi_{\Phi(a) V}^{\perp}=0 .
\end{gathered}
$$

To do this, we use the elementary fact that $\pi_{\Phi(a) V}=\Phi(a) \pi_{V} X=X^{\prime} \pi_{V} \Phi(a)^{*}$ and $\pi_{\Phi(a) V}^{\perp}=\Phi(a)^{-1 *} \pi_{V}^{\perp} Y=Y^{\prime} \pi_{V}^{\perp} \Phi(a)^{-1}$, where $X, X^{\prime}, Y, Y^{\prime}$ are matrix valued functions (of $z$ ). (For example, the first of these is proved by observing that $\Phi(a)^{-1} \pi_{\Phi(a) V}$ has image $V$, hence it may be written in the form $\pi_{V} X$.) Thus

$$
\begin{aligned}
\pi_{\Phi(a) V}^{\perp} & {\left[Z-\frac{1}{2}(1-1 / a) A\right] \pi_{\Phi(a) V} } \\
= & \pi_{\Phi(a) V}^{\perp}\left[-\frac{1}{2}(1-1 / a) A \Phi(a)+Z \Phi(a)\right] \pi_{V} X \\
= & \pi_{\Phi(a) V}^{\perp} \Phi(a) Z \pi_{V} X
\end{aligned}
$$

(as $\left.Z \Phi(a)=Z \cdot \Phi(a)+\Phi(a) Z=\frac{1}{2}(1-1 / a) A \Phi(a)+\Phi(a) Z\right)$. This is $Y^{\prime} \pi_{V}^{\perp} Z \pi_{V} X$, which is zero as $Z \pi_{V}=Z \cdot \pi_{V}+\pi_{V} Z=\pi_{V} Z$. Similarly,

$$
\pi_{\Phi(a) V}\left(Z-\frac{1}{2}(1-\bar{a}) A\right) \pi_{\Phi(a) V}^{\perp}=\pi_{\Phi(a) V} \Phi(1 / \bar{a}) Z \pi_{V}^{\perp} Y=X^{\prime} \pi_{V} Z \pi_{V}^{\perp} Y=0 .
$$

In fact the formula $g \cdot \Phi=\Psi \Phi g^{-1}$ holds whether or not the extended solution $\Phi$ is based (Theorem 5.3 of [Uh]), although this does not follow from Theorems 5.3 and 5.4 above. 
We conclude with a brief remark on possible generalizations. The most general $\Lambda_{I}$-valued function may be written $\Psi=\left(\Psi_{0}, \Psi_{\infty}\right)$, where $\Psi_{0}: D \times I(\varepsilon) \rightarrow$ $G l_{n} \mathbb{C}$ and $\Psi_{\infty}: D \times I(1 / \varepsilon) \rightarrow G l_{n} \mathbb{C}$. If $\Psi \Phi$ satisfies the linear equations where it is defined (i.e., in neighborhoods of the circles $C(\varepsilon), C(1 / \varepsilon)$ ), then so do $\Psi_{0} \Phi$ and $\Psi_{\infty} \Phi$. If $\Psi_{0}, \Psi_{\infty}$ extend to $\Lambda_{I}$-valued functions, for example, if $\Psi_{0}, \Psi_{\infty}$ are rational $G l_{n} \mathbb{C}$-valued functions on $S^{2}$ with no poles or singularities at $0, \infty$, then $\Psi_{0} \Phi \Psi_{0}(*)^{-1}$ and $\Psi_{\infty} \Phi \Psi_{\infty}(*)^{-1}$ are based extended solutions. They necessarily agree with the based extended solution $\left(\Psi_{0}, \Psi_{\infty}\right) \Phi\left(\Psi_{0}(*), \Psi_{\infty}(*)\right)^{-1}$, since all three are holomorphic on $\mathbb{C}^{*}$ and agree on some open sets. So in this case nothing new is obtained by considering $\left(\Psi_{0}, \Psi_{\infty}\right) \cdot \Phi$ rather than $\Psi_{0} \cdot \Phi$ or $\Psi_{\infty} \cdot \Phi$. It remains, however, to investigate the condition " $\Psi \Phi$ satisfies the linear equations" in the case where $\Psi$ is not given by $G l_{n} \mathbb{C}$-valued rational functions.

\section{Completion}

The limitations of the dressing pseudo-action for harmonic maps $S^{2} \rightarrow U_{n}$ are illustrated by the fact that energy is preserved (Theorem 4.5). For it is known [At] that the space of basepoint preserving holomorphic maps $S^{2} \rightarrow \Omega U_{n}$ of fixed degree is a finite-dimensional (noncompact) complex manifold, hence, by Propositions 1.1 and 1.3, the space of harmonic maps $S^{2} \rightarrow U_{n}$ of fixed energy is contained in a finite-dimensional space. Thus, the orbits of the pseudoaction on harmonic maps $S^{2} \rightarrow U_{n}$ are necessarily finite dimensional. This "collapsing" of the pseudo-action suggests that the loop groop is too cumbersome an object to be of much use in the description of the space of harmonic maps. However, the inherently discontinuous nature of the pseudo-action gives rise to the possibility of "completion", by means of which an orbit may be enlarged, as we shall now explain.

We shall consider in this section only the polar pseudo-action of $\S 5$. Let $\left\{g_{t}\right\}$ be a curve in $\Lambda_{I}$ and let $\Phi$ be an extended solution. If $\lim _{t \rightarrow 0} g_{t}=g$ but $\lim _{t \rightarrow 0} g_{t} \cdot \Phi \neq g \cdot \Phi$, then the extended solution $\widetilde{\Phi}=\lim _{t \rightarrow 0} g_{t} \cdot \Phi$ is not, a priori, obtained by applying a dressing transformation to $\Phi$. We shall refer to the procedure of obtaining $\widetilde{\Phi}$ from $\Phi$ as completion.

Example 6.1 (cf. $\S 12$ of [Uh]). Let

$$
g(\lambda)=\pi_{V}^{\perp}+\xi(\lambda) \pi_{V}=\pi_{V}^{\perp}+\frac{(\lambda-a)(1-1 / \bar{a})}{(\lambda-1 / \bar{a})(1-a)} \pi_{V}
$$

as in Example 5.5. If $a \neq 0, \infty$, then $g \in \Lambda_{I}$. By 5.5 we have

$$
g \cdot \Phi=\Psi \Phi g^{-1}, \quad \text { where } \Psi(z)(\lambda)=\pi_{\Phi(z)(a) V}^{\perp}+\frac{(\lambda-a)(1-1 / \bar{a})}{(\lambda-1 / \bar{a})(1-a)} \pi_{\Phi(z)(a) V} .
$$

Let us assume that $\lim _{a \rightarrow 0} \Phi(z)(a) V\left(=q(z)\right.$, say) exists in $\mathrm{Gr}_{k}\left(\mathbb{C}^{n}\right)$ for all $z$. Then we have

$$
\lim _{a \rightarrow 0} g \cdot \Phi(\lambda)=\left(\pi_{q}^{\perp}+\lambda \pi_{q}\right) \Phi(\lambda)\left(\pi_{V}^{\perp}+\frac{1}{\lambda} \pi_{V}\right)
$$


whereas

$$
\left(\lim _{a \rightarrow 0} g\right) \cdot \Phi(\lambda)=\left(\pi_{V}^{\perp}+\lambda \pi_{V}\right) \cdot \Phi(\lambda)=\Phi(\lambda)\left(\pi_{V}^{\perp}+\frac{1}{\lambda} \pi_{V}\right)
$$

since $\pi_{V}^{\perp}+\lambda \pi_{V}$ is in $\Lambda_{E}$. Hence the completion procedure potentially yields a "new" extended solution $\widetilde{\Phi}(\lambda)=\left(\pi_{q}^{\perp}+\lambda \pi_{q}\right) \Phi(\lambda)\left(\pi_{V}^{\perp}+(1 / \lambda) \pi_{V}\right)$.

Example 6.2. As a concrete case of 6.1, let us take the extended solution $\Phi$ to be $\Phi(z)(\lambda)=\pi_{f(z)}^{\perp}+\lambda \pi_{f(z)}$, where $f: S^{2} \rightarrow \mathrm{Gr}_{k}\left(\mathbb{C}^{n}\right)$ is a holomorphic map (as in $\S 1)$. We have to find $\lim _{a \rightarrow 0} \Phi(z)(a) V$. It is easy to see that the limit exists in the following two cases:

(a) If $V \subseteq f(z)$ for all $z$, then $\lim _{a \rightarrow 0} \Phi(z)(a) V=V$.

(b) If $V \cap f(z)=\{0\}$ for all $z$, then $\lim _{a \rightarrow 0} \Phi(z)(a) V=\pi_{f(z)}^{\perp} V$.

(If (a) or (b) does not hold, the limit does not exist; see Lemma 6.3 below.) In the first case we obtain $\widetilde{\Phi}=\Phi$, but in the second case we obtain

$$
\widetilde{\Phi}(\lambda)=\left(\pi_{q}^{\perp}+\lambda \pi_{q}\right)\left(\pi_{f}^{\perp}+\lambda \pi_{f}\right)\left(\pi_{V}^{\perp}+\frac{1}{\lambda} \pi_{V}\right),
$$

where $q=\pi_{f}^{\perp} V$. Now, $q(z)$ and $f(z)$ are orthogonal, and $q \oplus f=\pi_{f}^{\perp} V \oplus f=$ $\pi_{V}^{\perp} f \oplus V$, so this expression simplifies to

$$
\widetilde{\Phi}(\lambda)=\pi_{\pi_{V}^{\perp} f}^{\perp}+\lambda \pi_{\pi_{V}^{\perp} f} .
$$

It may be verified directly that $\tilde{\Phi}$ is an extended solution in this case; in fact the map $z \mapsto \pi_{V}^{\perp} f(z)$ is obviously a holomorphic map from $S^{2}$ to $\mathrm{Gr}_{k}\left(\mathbb{C}^{n}\right)$.

A first generalization of 6.2 is obtained on replacing $\Phi(\lambda)=\pi_{f}^{\perp}+\lambda \pi_{f}$ by any extended solution of the form $\Phi(\lambda)=A_{0}+\lambda A_{1}+\cdots+\lambda^{r} A_{r}$ (with $A_{i}: D \rightarrow$ $\left.\mathfrak{g l}_{n} \mathbb{C}\right)$. As in Example 6.1, one must compute $\lim _{a \rightarrow 0} \Phi(a) V$. This limit exists (in $\mathrm{Gr}_{k}\left(\mathbb{C}^{n}\right)$ ) if and only if the set $\left\{\lim _{a \rightarrow 0} \Phi(a)(\mathbb{C} v) \mid v \in V\right\}$ is a $k$-plane. Whether this is so may be determined from the next lemma.

Lemma 6.3. Let $V$ be a subspace of $\mathbb{C}^{n}$, and let $A_{0}, A_{1}, \ldots, A_{r} \in \mathfrak{g l}_{n} \mathbb{C}$. Put $A=A_{0}+a A_{1}+\cdots+a^{r} A_{r}$. Then

$$
\left\{\lim _{a \rightarrow 0} A(\mathbb{C} v) \mid v \in V\right\}=A_{0} V \cup A_{1}\left(\operatorname{Ker} A_{0} \cap V\right) \cup \cdots \cup A_{r}\left(\bigcap_{i=0}^{r-1} \operatorname{Ker} A_{i} \cap V\right) .
$$

Hence $\lim _{a \rightarrow 0} A V$ is a subspace if and only if one term in this union contains all the other terms.

Proof. Let $v \in V$. If $A_{i} v=0$ for all $i$, then $\lim _{a \rightarrow 0} A(\mathbb{C} v)=\{0\}$ for all $i$ and there is no contribution to the limit. So let us assume that for some $s \in\{0,1, \ldots, r\}$, one has $A_{i} v=0$ for $0 \leq i \leq s$ and $A_{s+1} v \neq 0$. Then $\lim _{a \rightarrow 0} A(\mathbb{C} v)=\lim _{a \rightarrow 0}\left(a^{s+1} A_{s+1}+\cdots+a^{r} A_{r}\right)(\mathbb{C} v)=A_{s+1}(\mathbb{C} v)$. The contribution to the limit from all such $v$ is therefore $A_{s+1}\left(\bigcap_{i=0}^{s} \operatorname{Ker} A_{i} \cap V\right)$. 
The case $r=1$ of this lemma was used in Example 6.2. Further applications of it will be postponed to $\S 7$.

A second generalization is obtained on replacing $g=\pi_{V}^{\perp}+\xi \pi_{V}$ by a product $g=g_{r} \cdots g_{1}=\left(\pi_{V_{r}}^{\perp}+\xi_{r} \pi_{V_{r}}\right) \cdots\left(\pi_{V_{1}}^{\perp}+\xi_{1} \pi_{V_{1}}\right)$. Uhlenbeck observed (see Theorem 5.4 of [Uh]) that any $g \in \Lambda_{I}$ which is a real $G l_{n} \mathbb{C}$-valued rational function may be factored in this way. (The argument is by induction on the number of poles and singularities.) For any extended solution $\Phi, g \cdot \Phi=\left(g_{r} \cdots g_{1}\right) \cdot \Phi$ exists for all $z \in D$, since the result of successively applying each $g_{i}$ does. Hence we may consider the limit as each $a_{i} \rightarrow 0$. Let us introduce the notation $\mathbf{a}_{i}=\left(a_{1}, \ldots, a_{i}\right)$ and

$$
\tilde{V}_{i}\left(\mathbf{a}_{i}\right)=\tilde{V}_{i}\left(a_{1}, \ldots, a_{i}\right)=\left(g_{i-1} \cdots g_{1}\right) \cdot \boldsymbol{\Phi}\left(a_{i}\right) V_{i}
$$

(for $i=1$ this is taken to mean $\left.\widetilde{V}_{1}\left(a_{1}\right)=\Phi\left(a_{1}\right) V_{1}\right)$. Then we have (by Example $5.5)$ :

$$
\begin{aligned}
& \left(g_{r} \cdots g_{1}\right) \cdot \Phi=\left(\pi_{V_{r}\left(\mathbf{a}_{r}\right)}^{\frac{1}{2}}+\xi_{r} \pi_{\widetilde{V}_{r}\left(\mathbf{a}_{r}\right)}\right)\left(g_{r-1} \cdots g_{1}\right) \cdot \Phi\left(\pi_{V_{r}}^{\perp}+\xi_{r}^{-1} \pi_{V_{r}}\right) \\
& =\left(\pi \frac{1}{\widetilde{V}_{r}\left(\mathbf{a}_{r}\right)}+\xi_{r} \pi_{\widetilde{V}_{r}\left(\mathbf{a}_{r}\right)}\right) \cdots\left(\pi_{\widetilde{V}_{1}\left(\mathbf{a}_{1}\right)}^{\frac{1}{n_{1}}}+\xi_{1} \pi_{\widetilde{V}_{1}\left(\mathbf{a}_{1}\right)}\right) \\
& \text {. } \Phi\left(\pi_{V_{1}}^{\perp}+\xi_{1}^{-1} \pi_{V_{1}}\right) \cdots\left(\pi_{V_{r}}^{\perp}+\xi_{r}^{-1} \pi_{V_{r}}\right) .
\end{aligned}
$$

Assume now that the limits $\lim _{\mathbf{a}_{i} \rightarrow 0} \widetilde{V}_{i}\left(\mathbf{a}_{i}\right)=\widetilde{V}_{i}$ exist for all $i$. Then

$$
\begin{aligned}
\lim _{\mathbf{a}_{i} \rightarrow 0}\left(g_{r} \ldots g_{1}\right) \cdot \Phi(\lambda)= & \left(\pi_{\widetilde{V}_{r}}^{\frac{1}{2}}+\lambda \pi_{\widetilde{V}_{r}}\right)\left(\lim _{\mathbf{a}_{i-1} \rightarrow 0}\left(g_{r-1} \cdots g_{1}\right) \cdot \Phi(\lambda)\right)\left(\pi_{V_{r}}^{\perp}+(1 / \lambda) \pi_{V_{r}}\right) \\
= & \left(\pi \frac{1}{\widetilde{V}_{r}}+\lambda \pi_{\widetilde{V}_{r}}\right) \cdots\left(\pi \frac{1}{\widetilde{V}_{1}}+\lambda \pi_{\widetilde{V}_{1}}\right) \\
& \cdot \Phi(\lambda)\left(\pi_{V_{1}}^{\perp}+(1 / \lambda) \pi_{V_{1}}\right) \cdots\left(\pi_{V_{r}}^{\perp}+(1 / \lambda) \pi_{V_{r}}\right) .
\end{aligned}
$$

Here, $\widetilde{V}_{i}$ may be obtained inductively as follows:

$$
\begin{aligned}
\widetilde{V}_{i}= & \lim _{\mathbf{a}_{i} \rightarrow 0} \widetilde{V}_{i}\left(\mathbf{a}_{i}\right)=\lim _{\mathbf{a}_{i} \rightarrow 0}\left(g_{i-1} \cdots g_{1}\right) \cdot \Phi\left(a_{i}\right) V_{i} \\
= & \lim _{\mathbf{a}_{i} \rightarrow 0} g_{i-1} \cdot\left(g_{i-2} \cdots g_{1} \cdot \Phi\right)\left(a_{i}\right) V_{i} \\
= & \lim _{\mathbf{a}_{i} \rightarrow 0}\left(\pi_{\widetilde{V}_{i-1}\left(\mathbf{a}_{i-1}\right)}^{\perp}+\xi_{i-1}\left(a_{i}\right) \pi_{\widetilde{V}_{i-1}\left(\mathbf{a}_{i-1}\right.}\right)\left(g_{i-2} \cdots g_{1}\right) \\
& \cdot \Phi\left(a_{i}\right)\left(\pi_{V_{i-1}}^{\perp}+\xi_{i-1}\left(a_{i}\right)^{-1} \pi_{V_{i-1}}\right) V_{i} \\
= & \lim _{\mathbf{a}_{i-2}, a \rightarrow 0}\left(\pi_{\widetilde{V}_{i-1}}^{\perp}+a \pi_{\widetilde{V}_{i-1}}\right)\left(g_{i-2} \cdots g_{1}\right) \cdot \Phi(a)\left(\pi_{V_{i-1}}^{\perp}+(1 / a) \pi_{V_{i-1}}\right) V_{i}
\end{aligned}
$$

(here we are using the fact that $\xi_{i-1}\left(a_{i}\right)=a_{i}-a_{i-1}+o\left(a_{i}, a_{i-1}\right)$ ).

The previous paragraph applies to any extended solution $\Phi$. In the particular case of $\Phi(\lambda)=\pi_{f}^{\perp}+\lambda \pi_{f}$, we can obtain an explicit result (Theorem 6.5 below). In doing so we shall make use of the following elementary fact (which was used already in Example 6.2): 
Lemma 6.4. Let $A, B$ be any two subspaces of $\mathbb{C}^{n}$. Then

$$
\left(\pi_{\pi_{A}^{\perp} B}^{\perp}+\lambda \pi_{\pi_{A}^{\perp} B}\right)\left(\pi_{A}^{\perp}+\lambda \pi_{A}\right)\left(\pi_{B}^{\perp}+(1 / \lambda) \pi_{B}\right)=\pi_{\pi_{B}^{\perp} A}^{\perp}+\lambda \pi_{\pi_{B}^{\perp} A} .
$$

Proof. This is equivalent to the statement $\pi_{A}^{\perp} B \oplus A=\pi_{B}^{\perp} A \oplus B$ (which is true because both sides are equal to the span of $A$ and $B$ ).

Theorem 6.5. Assume that the subspaces $V_{1}, \ldots, V_{r}$ satisfy the conditions $V_{i+1} \cap$ $\pi_{V_{i}}^{\perp} \cdots \pi_{V_{1}}^{\perp} f(z)=\{0\}, i=0,1, \ldots, r-1$. Then

$$
\lim _{\mathbf{a} \rightarrow 0} g_{r}(\lambda) \cdots g_{1}(\lambda) \cdot\left(\pi_{f}^{\perp}+\lambda \pi_{f}\right)=\pi_{W}^{\perp}+\lambda \pi_{W},
$$

where $W=\pi_{V_{r}}^{\perp} \cdots \pi_{V_{1}}^{\perp} f$.

Proof. Let $W_{i}=\pi_{V_{i}}^{\perp} \cdots \pi_{V_{1}}^{\perp} f$ (and $W_{0}=f$ ). We shall prove inductively the assertions

$$
\begin{gathered}
\tilde{V}_{i+1}=\pi_{W_{i}}^{\perp} V_{i+1}, \\
\lim _{\mathbf{a}_{i} \rightarrow 0} g_{i}(\lambda) \cdots g_{1}(\lambda) \cdot\left(\pi_{f}^{\perp}+\lambda \pi_{f}\right)=\pi_{W_{i}}^{\perp}+\lambda \pi_{W_{i}} .
\end{gathered}
$$

To start the induction, $\left(\mathrm{II}_{0}\right)$ is trivially true and $\left(\mathrm{I}_{0}\right)$ is true by Example 6.1. For the inductive step, assume $\left(\mathrm{I}_{i}\right)$ and $\left(\mathrm{II}_{i}\right)$.

To prove $\left(\mathrm{I}_{i+1}\right)$, we have

$$
\begin{aligned}
\widetilde{V}_{i+2} & =\lim _{\mathbf{a}_{i+2} \rightarrow 0} \tilde{V}_{i+2}\left(a_{i+2}\right) \\
& =\lim _{\mathbf{a}_{i}, a \rightarrow 0}\left(\pi_{\widetilde{V}_{i+1}}^{\perp}+a \pi_{\widetilde{V}_{i+1}}\right)\left(g_{i} \cdots g_{1}\right) \cdot\left(\pi_{f}^{\perp}+a \pi_{f}\right)\left(\pi_{V_{i+1}}^{\perp}+(1 / a) \pi_{V_{i+1}}\right) V_{i+2}
\end{aligned}
$$

by the discussion above. Now, $\tilde{V}_{i+1}=\pi_{W_{i}}^{\perp} V_{i+1}$ by $\left(\mathrm{I}_{i}\right)$, and $\lim _{\mathbf{a}_{i} \rightarrow 0}\left(g_{i} \cdots g_{1}\right)$ $\cdot\left(\pi_{f}^{\perp}+a \pi_{f}\right)=\pi_{W_{i}}^{\perp}+\lambda \pi_{W_{i}}$ by $\left(\mathrm{II}_{i}\right)$. Applying Lemma 6.4 with $A=W_{i}, B=V_{i+1}$ we find

$$
\begin{aligned}
\tilde{V}_{i+2} & =\lim _{a \rightarrow 0}\left(\pi_{\pi_{B}^{\perp} A}^{\perp}+a \pi_{\pi_{B}^{\perp} A}\right) V_{i+2} \\
& =\lim _{a \rightarrow 0}\left(\pi_{W_{i+1}}^{\perp}+a \pi_{W_{i+1}}\right) V_{i+2}=\pi_{W_{i+1}^{\perp}}^{\perp} V_{i+2}
\end{aligned}
$$

by the same argument as in Example 6.2 (i.e., by the case $r=1$ of Lemma 6.3).

To prove $\left(\mathrm{II}_{i+1}\right)$ we have

$$
\begin{aligned}
& \lim _{\mathbf{a}_{i+1} \rightarrow 0} g_{i+1}(\lambda) \cdots g_{1}(\lambda) \cdot\left(\pi_{f}^{\perp}+\lambda \pi_{f}\right) \\
& \quad=\left(\pi \widetilde{V}_{i+1}^{\perp}+\lambda \pi \widetilde{V}_{\widetilde{V}_{i+1}}\right)\left(\lim _{\mathbf{a}_{i} \rightarrow 0} g_{i}(\lambda) \cdots g_{1}(\lambda) \cdot\left(\pi_{f}^{\perp}+\lambda \pi_{f}\right)\right)\left(\pi_{V_{i+1}}^{\perp}+(1 / \lambda) \pi_{V_{i+1}}\right) \\
& \quad=\left(\pi \widetilde{V}_{i+1}^{\perp}+\lambda \pi_{\widetilde{V}_{i+1}}\right)\left(\pi_{W_{i}}^{\perp}+\lambda \pi_{W_{i}}\right)\left(\pi_{V_{i+1}}^{\perp}+(1 / \lambda) \pi_{V_{i+1}}\right)
\end{aligned}
$$


on using $\left(\mathrm{II}_{i}\right)$. By $\left(\mathrm{I}_{i}\right), \widetilde{V}_{i+1}=\pi_{W_{i}}^{\perp} V_{i+1}$. So by Lemma 6.4 we obtain finally $\pi_{W_{i+1}}^{\perp}+\lambda \pi_{W_{i+1}}$. This completes the inductive step.

Although the conditions on $V_{1}, \ldots, V_{r}$ appear rather stringent, this theorem covers all cases. For, if the limit exists, and the conditions hold for $i=1, \ldots, j-1$, we must have either $V_{j+1} \cap \pi_{V_{j}}^{\perp} \cdots \pi_{V_{1}}^{\perp} f(z)=\{0\}$ or $V_{j+1} \subseteq$ $\pi_{V_{j}}^{\perp} \cdots \pi_{V_{1}}^{\perp} f(z)$, and in the latter case we obtain $\widetilde{V}_{j+1}=V_{j+1}$.

\section{RELATIONS WITH KNOWN RESULTS ON HARMONIC MAPS}

Harmonic maps from $S^{2}$ to $U_{n}$ have been classified (see [Se, Uh, Va, Wo]) in a sense that will now be described. First, one has the procedure of "adding a uniton":

Theorem 7.1 [Uh, Theorem 12.1]. Let $\Phi$ be a real extended solution. Let $f: S^{2} \rightarrow \mathrm{Gr}_{k}\left(\mathbb{C}^{n}\right)$ be a (not necessarily holomorphic) map, and define $\Psi(z)(\lambda)=$ $\pi_{f(z)}^{\perp}+\lambda \pi_{f(z)}$. Then $\Psi \Phi$ is an extended solution if and only if the equations

$$
\pi_{f}^{\perp} A \pi_{f}=0, \quad \pi_{f}\left(Z-\frac{1}{2} A\right) \pi_{f}^{\perp}=0
$$

are satisfied, where $A$ is the result of evaluating $Z \cdot \Phi \Phi^{-1}$ at $\lambda=-1$.

Proof. Similar to the proof of Theorem 5.4.

This can be used to obtain the classification theorem for real extended solutions on $S^{2}$ :

Theorem 7.2 [Uh, Theorem 14.6; Va, Corollaries 7, $7^{\prime}$; Se]. Let $\Phi$ be a real extended solution on $S^{2}$. Then there exists a loop $g \in \Omega U_{n}$ and maps $f_{1}, \ldots, f_{k}$ with $f_{i}: S^{2} \rightarrow \mathrm{Gr}_{k_{i}}\left(\mathbb{C}^{n}\right)$, where $k \leq n-1$, such that

(1) $\Phi(\lambda)=\left(\pi_{f_{k}}^{\perp}+\lambda \pi_{f_{k}}\right) \cdots\left(\pi_{f_{1}}^{\perp}+\lambda \pi_{f_{1}}\right) g(\lambda)$.

(2) If $\Phi_{i}(\lambda)$ denotes $\left(\pi_{f_{i}}^{\perp}+\lambda \pi_{f_{i}}\right) \cdots\left(\pi_{f_{1}}^{\perp}+\lambda \pi_{f_{1}}\right) g(\lambda)$, then $\Phi_{i}$ is obtained from $\Phi_{i-1}$ by adding a uniton.

This may be interpreted as saying that a general harmonic map $\phi$ is constructed from holomorphic data, since (a) the first map $f_{1}: S^{2} \rightarrow \mathrm{Gr}_{k_{1}}\left(\mathbb{C}^{n}\right)$ is necessarily holomorphic (as $\pi_{f_{1}}^{\perp}+\lambda \pi_{f_{1}}$ is an extended solution, cf. $\S 1$ ), and (b) the second of the equations for adding a uniton in Theorem 7.1 says that $f$ is holomorphic with respect to the complex structure whose $\bar{\partial}$-operator is $\bar{Z}+\frac{1}{2} A$. A more explicit version of this reduction to holomorphic data has been given by Wood [Wo].

In this section we shall comment on the relevance of the polar pseudo-action to Theorems 7.1 and 7.2. The underlying problem, as we understand it, is whether the pseudo-action is of any importance in describing the "moduli space" 
of harmonic maps. Uhlenbeck made the interesting suggestion ( $\S 12$ of [Uh]) that adding a uniton should arise through the completion process described in $\S 6$, i.e., as a limit of the pseudo-action. Some evidence for this is provided by the fact that the equations for adding a uniton (see Theorem 7.1) arise by taking the limit as $a \rightarrow 0$ of the pseudo-action equations

$$
\pi_{f}^{\perp}\left(Z-\frac{1}{2}(1-1 / a) A\right) \pi_{f}=0, \quad \pi_{f}\left(Z-\frac{1}{2}(1-\bar{a}) A\right) \pi_{f}^{\perp}=0
$$

(see Theorem 5.4 and the following discussion). Example 6.1 appears to illustrate this, as it seems to show that $\pi_{q}^{\perp}+\lambda \pi_{q}$ is "added" to the extended solution $\Phi$. However, this addition is an illusion. For example, let $\Phi(\lambda)=\pi_{f}^{\perp}+\lambda \pi_{f}$ (Example 6.2). Then $q(z)$ is either $V$ or $\pi_{f(z)}^{\perp} V$, and so $\left(\pi_{q}^{\perp}+\lambda \pi_{q}\right)\left(\pi_{f}^{\perp}+\lambda \pi_{f}\right)$ is either $\left(\pi_{f}^{\perp}+\lambda \pi_{f}\right)\left(\pi_{V}^{\perp}+\lambda \pi_{V}\right)$ or $\left(\pi_{\pi_{V}^{\perp} f}^{\perp}+\lambda \pi_{\pi_{V} f}\right)\left(\pi_{V}^{\perp}+\lambda \pi_{V}\right)$. In each case, the second factor is just a constant loop, and nothing is actually "added". More generally:

Lemma 7.3. Let $\Phi$ be an extended solution of the form

$$
\Phi(z)(\lambda)=\sum_{i=-k}^{l} \lambda^{i} A_{i}(z) .
$$

Let $g \in \Lambda_{I}$, and assume that $g \cdot \Phi$ exists for all $z$. Then $g \cdot \Phi$ is of the form $g \cdot \Phi(z)(\lambda)=\sum_{i=-k}^{l} \lambda^{i} B_{i}(z)$.

Proof. By $\S 5$ we have $g \cdot \Phi=\Psi \Phi g^{-1}$, where $\Psi$ is $\Lambda_{I}$-valued (for each $z$ ). Since $\Psi, g^{-1}$ are holomorphic and $G l_{n} \mathbb{C}$-valued at $\lambda=0, \infty$, it follows that the poles of $g \cdot \Phi$ at 0 and $\infty$ have order less than or equal to those of $\Phi$.

Proposition 7.4. Consider the real extended solution $\Phi(\lambda)=\pi_{f}^{\perp}+\lambda \pi_{f}$, where $f: S^{2} \rightarrow \mathrm{Gr}_{k}\left(\mathbb{C}^{n}\right)$ is a nonconstant holomorphic map. Then the completion procedure never gives rise to a constant extended solution $\widetilde{\Phi}(\lambda)=h(\lambda), h \in \Omega U_{n}$. Conversely, starting with a constant extended solution $\Phi=h$, it is impossible to produce by completion an extended solution of the form $\widetilde{\Phi}(\lambda)=\pi_{f}^{\perp}+\lambda \pi_{f}$, where $f$ is a nonconstant holomorphic map.

Proof. Let $\left\{g_{t}\right\}$ be a family of elements of $\Lambda_{I}$. By Lemma 7.3, $g_{t}(\lambda) \cdot\left(\pi_{f}^{\perp}+\lambda \pi_{f}\right)$ is necessarily of the form $\pi_{f_{t}}^{\perp}+\lambda \pi_{f_{t}}$ (the lemma may be applied both to $g_{t}$ and to $g_{t}^{-1}$ ). Since the action preserves energy, $\left\{f_{t}\right\}$ is a family of nonconstant holomorphic maps into a Grassmannian. Such a family of holomorphic maps can never have a constant map as a limit. The converse is trivial, as $g_{t} \cdot h$ is always independent of $z$.

It is clear that the pseudo-action by a family of elements of $\Lambda_{E}$ is equally useless (in passing between $\pi_{f}^{\perp}+2 \pi_{f}$ and $h$ ), although we have not ruled out the possibility that some more general completion procedure, perhaps by a family not contained in $\Lambda_{E} \Lambda_{I}$, might work. 
Now we shall obtain more detailed information in the case of harmonic maps from $S^{2}$ to $\mathbb{C} P^{n}$. In this situation, harmonic maps (and the corresponding extended solutions) have a straightforward explicit description in terms of holomorphic maps. Indeed, any harmonic map $f: S^{2} \rightarrow \mathbb{C} P^{n}$ is known to be of the form $f(z)=g_{i}(z)^{\perp} \cap g_{i+1}(z)$, where $g_{i}: S^{2} \rightarrow \mathrm{Gr}_{i+1}\left(\mathbb{C}^{n+1}\right)$ denotes the $i$ th "associated curve" of some holomorphic map $g: S^{2} \rightarrow \mathbb{C} P^{n}$. (In this situation, both $g_{i}$ and $g_{i+1}$ are holomorphic maps.) It is easy to verify that an extended solution corresponding to the harmonic map $f$ is

$$
\left(\pi_{g_{i}}^{\perp}+\lambda \pi_{g_{i}}\right)\left(\pi_{g_{i+1}}^{\perp}+\lambda \pi_{g_{i+1}}\right) \text {, }
$$

which we shall denote simply by $\left(\pi_{i}^{\perp}+\lambda \pi_{i}\right)\left(\pi_{i+1}^{\perp}+\lambda \pi_{i+1}\right)$ in order to simplify notation. In [Gul] this description of harmonic maps $S^{2} \rightarrow \mathbb{C} P^{n}$ was cast in a more algebraic form by noting that $g_{i}$ may be expressed as $P \phi_{i}$, where $\phi_{i}: S^{2} \rightarrow \mathrm{Gr}_{i+1}\left(\mathbb{C}^{N+1}\right)$ is given by the orbit of the space spanned by the first $i+1$ weight vectors of the standard irreducible representation $S^{N} \lambda$ of $S U_{2}$, and where $P$ is some (possibly singular) $(N+1) \times(N+1)$ matrix. Thus, a harmonic map $f=\left(P \phi_{i}\right)^{\perp} \cap\left(P \phi_{i+1}\right)$ is specified by three pieces of information: an irreducible representation of $S U_{2}$, an integer $i$, and a matrix $P$.

Although one cannot hope to describe the set of harmonic maps $S^{2} \rightarrow \mathbb{C} P^{n}$ as the result of "completing" a single orbit of the polar pseudo-action, it is nevertheless of interest to see what operations on such harmonic maps do in fact arise from the completion procedure. To answer this, we shall begin with an example (the promised application of Lemma 6.3).

Example 7.5. We shall compute

$$
\lim _{a \rightarrow 0} g \cdot\left(\pi_{i}^{\perp}+\lambda \pi_{i}\right)\left(\pi_{i+1}^{\perp}+\lambda \pi_{i+1}\right),
$$

where $g=\pi_{V}^{\perp}+\xi \pi_{V}$ as in $\S 6$. We obtain

$$
\left(\pi_{\tilde{V}}^{\frac{1}{\tilde{V}}}+\lambda \pi_{\widetilde{V}}\right)\left(\pi_{i}^{\perp}+\lambda \pi_{i}\right)\left(\pi_{i+1}^{\perp}+\lambda \pi_{i+1}\right)\left(\pi_{V}^{\perp}+(1 / \lambda) \pi_{V}\right),
$$

where $\tilde{V}=\lim _{a \rightarrow 0}\left(\pi_{i}^{\perp}+a \pi_{i}\right)\left(\pi_{i+1}^{\perp}+a \pi_{i+1}\right) V$. Applying Lemma 6.3 with $A_{0}=$ $\pi_{i+1}^{\perp}, A_{1}=\pi_{i}^{\perp} \pi_{i+1}$, and $A_{2}=\pi_{i}$, we find three cases. (Note that, since the images of $A_{0}, A_{1}, A_{2}$ are orthogonal here, the limit exists if and only if two of the terms in the union in Lemma 6.3 are zero.)

Case 1. $A_{0} V=A_{1}\left(\operatorname{Ker} A_{0} \cap V\right)=\{0\}$. The conditions are equivalent to $V \subseteq$ $g_{i}(z)$ for all $z$, and one finds $\widetilde{V}=V$. Hence the new extended solution is equal to the one we started with.

Case 2. $A_{0} V=A_{2}\left(\operatorname{Ker} A_{0} \cap \operatorname{Ker} A_{1} \cap V\right)=\{0\}$. The conditions are equivalent to $V \subseteq g_{i+1}(z), V \cap g_{i}(z)=\{0\}$, and so $\widetilde{V}=\pi_{i}^{\perp} V$. Since $V \subseteq g_{i+1}(z), \pi_{V}$ commutes with $\pi_{i+1}$. Hence, on applying Lemma 6.4 with $A=g_{i}(z), B=V$, we find the new extended solution to be

$$
\left(\pi_{\pi_{V}^{\perp} g_{i}}^{\perp}+\lambda \pi_{\pi_{V}^{\perp} g_{i}}\right)\left(\pi_{i+1}^{\perp}+\lambda \pi_{i+1}\right) .
$$


Case 3. $A_{1}\left(\operatorname{Ker} A_{0} \cap V\right)=A_{2}\left(\operatorname{Ker} A_{0} \cap \operatorname{Ker} A_{1} \cap V\right)=\{0\}$. The conditions are equivalent to $V \cap g_{i+1}(z)=\{0\}$, and so $\widetilde{V}=\pi_{i+1}^{\perp} V$. Since $\pi_{\pi_{i+1}^{\perp} V}$ commutes with $\pi_{i}$, we may apply Lemma 6.4 with $A=g_{i+1}(z), B=V$ to rewrite the new extended solution as

$$
\left(\pi_{i}^{\perp}+\lambda \pi_{i}\right)\left(\pi_{\pi_{\nu}^{\perp} g_{i+1}}^{\perp}+\lambda \pi_{\pi_{\nu}^{\perp} g_{i+1}}\right) .
$$

Now, the new extended solutions produced in Cases 2 and 3 do not in general correspond to harmonic maps into $\mathbb{C} P^{n}$. If we impose on $V$ the additional restriction that the extended solutions do correspond to maps into $\mathbb{C} P^{n}$, then the extended solutions are necessarily of the form $\left(P^{\perp}-P\right)\left(Q^{\perp}-Q\right)$ at $\lambda=-1$, where $P, Q$ are projection matrices with $P Q=Q P$ and $\operatorname{rank} Q=\operatorname{rank} P+1$. In Case 2 this forces the dimension of $V$ to be zero or one, since $V \oplus g_{i} \subseteq g_{i+1}$. If $\operatorname{dim} V=1$, then $V \oplus g_{i}=g_{i+1}$, hence $Z g_{i+1} \subseteq Z g_{i} \subseteq g_{i+1}$, i.e., $g_{i+1}$ is antiholomorphic. But $g_{i+1}$ is also holomorphic, hence constant. This forces $g_{i}$ to have degree zero, hence $g_{i}$ is constant too, which (by definition of $g_{i+1}$ ) gives $g_{i+1}=g_{i}$, a contradiction. Hence $V=\{0\}$. In Case 3 we must have $g_{i} \subseteq \pi_{V}^{\perp} g_{i+1}$, hence $g_{i}(z) \subseteq V^{\perp}$ and $g_{i}=\pi_{V}^{\perp} g_{i}$.

To summarize, we have shown that the operations on harmonic maps $S^{2} \rightarrow$ $\mathbb{C} P^{n}$ produced by the completion procedure $\Phi \mapsto \lim _{a \rightarrow 0} g \cdot \Phi$ are precisely the operations

$$
f=g_{i}^{\perp} \cap g_{i+1} \longmapsto\left(\pi_{V}^{\perp} g_{i}\right)^{\perp} \cap\left(\pi_{V}^{\perp} g_{i+1}\right),
$$

where $V$ is any subspace of $\mathbb{C}^{n+1}$ with $g_{i}(z) \subseteq V^{\perp}$ and $V \cap g_{i+1}(z)=\{0\}$ for all $z \in S^{2}$.

We can go further and investigate the operations on harmonic maps $S^{2} \rightarrow$ $\mathbb{C} P^{n}$ produced by the completion procedure $\Phi \mapsto \lim _{\mathbf{a}_{r} \rightarrow 0} g_{r} \cdots g_{1} \cdot \Phi$. A calculation analogous to that in Theorem 6.5 shows that these operations are simply the result of repeating operations of the type obtained in Example 7.5.

Examples 6.2 and 7.5 suggest a modification of the completion procedure of $\S 6$, in which we take a curve $\left\{g_{t}\right\}$ in $\Lambda_{I}$ and consider $\lim _{t \rightarrow 0} g_{t} \cdot \Phi$ as before, but in which we allow $\lim _{t \rightarrow 0} g_{t} \cdot \Phi$ to have a finite number of removable singularities. We obtain a "new" extended solution by removing these singularities. For example, we may modify 6.2 by taking a holomorphic map $f: S^{2} \rightarrow \mathrm{Gr}_{k}\left(\mathbb{C}^{n}\right)$ and a subspace $V \subseteq \mathbb{C}^{n}$ such that $V \cap f(z)=\{0\}$ except at $z=z_{1}, \ldots, z_{r}$. By (ordinary) completion we obtain the holomorphic map $\pi_{V}^{\perp} f$ on $S^{2}-\left\{z_{1}, \ldots, z_{r}\right\}$. The singularities $z_{1}, \ldots, z_{r}$ are removable, and the result of removing them gives a holomorphic map $S^{2} \rightarrow \mathrm{Gr}_{k}\left(\mathbb{C}^{n}\right)$ which in general will have lower energy (equivalently, lower degree) than $f$. By a suitable choice of $V$, the energy may be reduced to zero:

Lemma 7.6. Let $g: S^{2} \rightarrow \mathrm{Gr}_{k}\left(\mathbb{C}^{n}\right)$ be holomorphic. Then there exists an $(n-k)$ dimensional subspace $V$ of $\mathbb{C}^{n}$ such that $V \cap g(z)=\{0\}$ for all but a finite number of values of $z$. (Hence $\pi_{V}^{\perp} g$ defines a constant map into $\mathrm{Gr}_{k}\left(\mathbb{C}^{n}\right)$.) 
Proof. Fix some $z_{0} \in S^{2}$ and let $V=g\left(z_{0}\right)^{\perp}$. We claim that $\pi_{V}^{\perp} g(z)$ has dimension $k$ for all but a finite number of values of $z$ (which implies the required property of $V$ ). First, $\operatorname{dim} \pi_{V}^{\perp} g(z)=k$ for all $z$ in a neighborhood of $z_{0}$, by lower semicontinuity of $\operatorname{dim} \pi_{V}^{\perp} g$. But then this equation must hold for all but a finite number of $z$, by holomorphicity of $\pi_{V}^{\perp} g$.

Extending this, we have

Theorem 7.7. By applying the modified completion procedure twice, any harmonic map $S^{2} \rightarrow \mathbb{C} P^{n}$ may be reduced to a constant map.

Proof. Consider the extended solution

$$
\Phi(\lambda)=\left(\pi_{i}^{\perp}+\lambda \pi_{i}\right)\left(\pi_{i+1}^{\perp}+\lambda \pi_{i+1}\right)
$$

corresponding to a harmonic map $S^{2} \rightarrow \mathbb{C} P^{n}$. By Example 7.5, Case 3, we have

$$
\lim _{a \rightarrow 0} g \cdot \Phi(\lambda)=\left(\pi_{i}^{\perp}+\lambda \pi_{i}\right)\left(\pi_{\pi_{V}^{\perp} g_{i+1}}^{\perp}+\lambda \pi_{\pi_{V}^{\perp} g_{i+1}}\right),
$$

where $g(\lambda)=\pi_{V}^{\perp}+\xi \pi_{V}$. By Lemma 7.6 we may choose an $(n-i-1)$ dimensional subspace $V$ so that $\pi_{V}^{\perp} g_{i+1}$ is a constant subspace $U$ (and $V \cap$ $g_{i+1}(z)=\{0\}$ for all but a finite number of values of $\left.z\right)$. We obtain an extended solution of the form $\left(\pi_{i}^{\perp}+\lambda \pi_{i}\right)\left(\pi_{U}^{\perp}+\lambda \pi_{U}\right)$. The process may now be repeated with $\pi_{i}^{\perp}+\lambda \pi_{i}$.

We have primarily been discussing maps from $S^{2}$ into $\mathbb{C} P^{n}$ in this section, but the following problem now suggests itself:

Problem. Can any extended solution be reduced to a constant solution by repeated application of the modified completion procedure?

The method of 7.6, 7.7 applies directly to extended solutions of the form $\left(\pi_{f_{2}}^{\perp}+\lambda \pi_{f_{2}}\right)\left(\pi_{f_{1}}^{\perp}+\lambda \pi_{f_{1}}\right)$, where $f_{1}, f_{2}$ are holomorphic maps into Grassmannians and $f_{1}(z) \subseteq f_{2}(z)$ for all $z \in S^{2}$. In particular, the isotropic harmonic maps into Grassmannians constructed by Erdem and Wood [EW] correspond to extended solutions of this form, and hence may be reduced to constant maps by applying the modified completion procedure twice. On the other hand, this method does not extend directly to general 2 -unitons, as $\pi_{f_{2}}^{\perp}+\lambda \pi_{f_{2}}$ is not usually itself an extended solution.

\section{REFERENCES}

[AB] J. Avan and G. Bellon, Groups of dressing transformations for integrable models in dimension two, Phys. Lett. B 213 (1988), 459-465.

[AJS] G. Arsenault, M. Jacques, and Y. Saint-Aubin, Collapse and exponentiation of infinite symmetry algebras of Euclidean projective and Grassmannian sigma models, J. Math. Phys. 29 (1988), 1465-1471. 
[At] M. F. Atiyah, Instantons in two and four dimensions, Comm. Math. Phys. 93 (1984), 437451.

[EL] J. Eells and L. Lemaire, A report on harmonic maps, Bull. London Math. Soc. 10 (1978), $1-68$.

[EW] S. Erdem and J. C. Wood, On the construction of harmonic maps into a Grassmannian, J. London Math. Soc. (2) 28 (1983), 161-174.

[Gu1] M. A. Guest, Geometry of maps between generalized flag manifolds, J. Differential Geom. 25 (1987), 223-247.

[Gu2] _- Harmonic two-spheres in complex projective space and some open problems, Exposition. Math. (to appear).

[JS] M. Jacques and Y. Saint-Aubin, Infinite dimensional Lie algebras acting on the solution space of various sigma models, J. Math. Phys. 28 (1987), 2463-2479.

[Po] K. Pohlmeyer, Integrable Hamiltonian systems and interactions through constraints, Comm. Math. Phys. 46 (1976), 207-221.

[PS] A. N. Pressley and G. B. Segal, Loop groups, Oxford Univ. Press, 1986.

[Ro] Y. L. Rodin, The Riemann boundary value problem on Riemann surfaces, Reidel, Dordrecht, 1988.

[Se] G. B. Segal, Loop groups and harmonic maps, Advances in Homotopy Theory (S. M. Salamon, B. Steer, and W. A. Sutherland, eds.), LMS Lecture Notes 139, Cambridge Univ. Press, 1989, pp. 153-164.

[Uh] K. Uhlenbeck, Harmonic maps into Lie groups (Classical solutions of the chiral model), J. Differential Geom. 30 (1989), 1-50.

[Va] G. Valli, On the energy spectrum of harmonic 2-spheres in unitary groups, Topology 27 (1988), 129-136.

[Wi] G. Wilson, Infinite dimensional Lie groups and algebraic geometry in soliton theory, Philos. Trans. Roy. Soc. London Ser. A 315 (1985), 393-404.

[Wo] J. C. Wood, Explicit construction and parametrization of harmonic two-spheres in the unitary group, Proc. London Math. Soc. 58 (1989), 608-624.

[ZM] V. E. Zakharov and A. V. Mikhailov, Relativistically invariant two-dimensional models of field theory which are integrable by means of the inverse scattering problem method, Soviet Phys. JETP 47 (1978), 1017-1027.

[ZS] V. E. Zakharov and A. B. Shabat, Integration of non-linear equations of mathematical physics by the inverse scattering method. II, Functional Anal. Appl. 13 (1979), 13-22.

Department of Mathematics, University of Georgia, Athens, Georgia 30602

Current address: Department of Mathematics, University of Illinois, Urbana, Illinois 61801

(M. A. Guest) Department of Mathematics, University of Rochester, Rochester, New YORK 14627 